\title{
Discrete unified gas kinetic scheme for all Knudsen flows. III. Binary mixtures of Maxwell molecules
}

\author{
Yue Zhang, ${ }^{1}$ Lianhua Zhu, ${ }^{1}$ Ruijie Wang, ${ }^{2}$ and Zhaoli Guo ${ }^{1, *}$ \\ ${ }^{1}$ State Key Laboratory of Coal Combustion, \\ School of Energy and Power Engineering, \\ Huazhong University of Science and Technology, Wuhan 430074, China \\ ${ }^{2}$ School of Power and Energy, Northwestern Polytechnical University, Xi'an 710072, China
}

(Dated: November 22, 2019)

\begin{abstract}
Recently a discrete unified gas kinetic scheme (DUGKS) in finite-volume formulation based on the Boltzmann model equation is developed for gas flows in all flow regimes. The original DUGKS is designed for flows of single-species gases. In this work, we extend the DUGKS to flows of binary gas mixtures of Maxwell molecules based on the AAP kinetic model [P. Andries et al., J. Stat. Phys. 106, 993 (2002)]. A particular feature of the method is that the flux at each cell interface is evaluated based on the characteristic solution of the kinetic equation itself, thus the numerical dissipation is low in comparison with that using direct reconstruction. Furthermore, the implicit treatment of the collision term enable the time step to be free from the restriction of the relaxation time. Unlike the DUGKS for single-species flows, a nonlinear system must be solved to determine the interaction parameters appearing in the equilibrium distribution function, which can be obtained analytically for Maxwell molecules. Several tests are performed to validate the scheme, including the shock structure problem under different Mach numbers and molar concentrations, the channel flow driven by small gradient of pressure, temperature or concentration, the plane Couette flow, and the shear driven cavity flow under different mass ratios and molar concentrations. The results are compared with those from other reliable numerical methods. The results show that the proposed scheme is an effective and reliable method for binary gas mixtures in all flow regimes.
\end{abstract}

Keywords: binary gas mixtures, Maxwell molecules, Boltzmann model equation, kinetic scheme

\footnotetext{
* Corresponding author: zlguo@hust.edu.cn
} 


\section{INTRODUCTION}

Rarefied gas mixtures flows exist widely in nature and practical applications, such as chemical reactions, evaporation-condensation and the Micro-Electro-Mechanical-System (MEMS). The rarefaction degree of gas flows is normally characterized by the Knudsen number $(\mathrm{Kn})$, which is defined as the ratio of the mean free path of gas molecules to the characteristic length of the system. The conventional fluid dynamics models, such as the Euler equations and the Navier-Stokes equations, are valid for continuum flows $(\mathrm{Kn}<0.001)$, but for flows with relative large $\mathrm{Kn}$, non-equilibrium effects will appear and continuum models become invalid [1].

Alternatively, the Boltzmann equation can be used to describe the gas mixtures flows in all regimes. But it is difficult to obtain the accurate solutions of the Boltzmann equation directly due to the complicated collision term. Conventionally, the Direct Simulation Monte Carlo (DSMC) method was employed to investigate non-equilibrium behaviors of the rarefied gas mixtures in many studies, e.g., [2-5], which is a prevailing numerical technique for simulating moderate and highly rarefied gas flows. However, the streaming and collision processes of the DSMC are decoupled, such that the time step and mesh size are limited by the molecular collision time and the mean free path, respectively [6]. This limitation leads to expensive computational costs for continuum and near-continuum flows. It is noted that some efforts have been made to reduce these difficulties [7, 8]. Besides the DSMC method, some deterministic numerical methods for the Boltzmann equation, such as [9-12], have been applied to gas mixtures flows with simple geometries. These deterministic methods can offer accurate solutions of the full Boltzmann equation, but are usually rather complicated and computationally expensive.

Some efforts have been devoted to simplify the full Botlzmann equation for gas mixtures by replacing the full collision operator with certain simplified models. Compared with the single-species kinetic model equations, the non-unitary mass ratio between different molecular species increases the difficulty. One of such models is the McCormack model [13] which linearizes the nonlinear collision term under the assumption that the systems only slight deviate from equilibrium; it is noted that extension to nonlinear problems has also been made recently $[14,15]$. Another simplified model is the so called AAP model [16] in which the collision term is modeled by a single Bhatnagar-Gross-Krook (BGK) [17] operator con- 
sidering both self-collision and cross-collision effects. Owing to its simple formulation, the AAP model has been applied to a number of rarefied mixture flows [18-21].

Based on the kinetic models, some numerical schemes have been developed, such as the lattice Boltzmann method (LBM) [22-24] and the discrete velocity methods (DVM) [25-27]. Particularly, a unified gas kinetic scheme (UGKS) for binary gas mixtures of hard sphere molecules and Maxwell molecules has been constructed [28, 29] for all flow regimes based on the AAP model. The original UGKS is designed for single-species gas flows covering different flow regimes [30,31], which is a finite-volume scheme for the discrete velocity Boltzmann model equation. A distinctive feature of the UGKS is that the reconstruction of the numerical flux is based on the local analytical characteristic solution of the kinetic equation rather than interpolation, such that the numerical dissipation is small. Furthermore, the semi-implicit discretization of the collision term in UGKS enables it to be unified stable, i.e., the time step is not limited by the mean-collision time. The UGKS also has the nice asymptotic preserving (AP) property [32], i.e., it solves the Navier-Stokes equations in the continuum flow regime.

Recently, another unified kinetic method, i.e., the discrete unified gas kinetic scheme (DUGKS) $[33,34]$ was developed for single-species gas flows covering different flow regimes. The DUGKS shares all the advantages of the UGKS, but some apparent differences also exist between the two schemes. Firstly, they achieve the characteristic solution by different approach: the UGKS uses an analytical temporal-spatial integral solution of the governing equation, while the DUGKS uses a discrete characteristic solution which is much simpler than the analytical integral one. Secondly, in the UGKS, flow variables are required to be updated first to evaluate explicitly the implicit treatment of the collision term, while the DUGKS removes the implicitness by introducing a new distribution function that is tracked in implementation. The above differences make the DUGKS more efficient than the UGKS [28]. Compared with the LBM, the computational cost of the DUGKS is somewhat expensive with the same uniform mesh, but is less expensive if a nonuniform mesh is employed [35]. The DUGKS has already been applied successfully to flows of single-species gases from continuum to rarefied regimes [36-39]. Recently, some extensions of DUGKS to complex flows have also been made. For example, a DUGKS for two-phase flows was proposed based on the phase-field theory [40]. Another possible extension is for flows in porous media, by making use of unstructured meshes like the finite-volume LBM [41, 42]. 
The aim of this work is to extend the DUGKS to binary gas mixtures of Maxwell molecules based on the AAP model. The remaining part of this paper is organized as follows. Section 2 will introduce the AAP model for binary gas mixtures. In Sec. 3, the DUGKS will be constructed based on the AAP model, and in Sec. 4 several numerical tests are performed. Finally, a brief summary is given in Sec. 5 .

\section{THE AAP MODEL FOR GAS MIXTURES}

The Boltzmann equation for a binary gas mixture of species $A$ and $B$ can be written as [43],

$$
\frac{\partial f_{\alpha}}{\partial t}+\boldsymbol{\xi} \cdot \nabla f_{\alpha}=Q_{\alpha}(f, f)
$$

with

$Q_{\alpha}(f, f)=\sum_{\alpha=A, B} Q_{\alpha \beta}\left(f_{\alpha}, f_{\beta}\right), \quad Q_{\alpha \beta}\left(f_{\alpha}, f_{\beta}\right)=\int_{R^{3}} \int_{B_{+}}\left(f_{\alpha}^{\prime} f_{\beta^{*}}^{\prime}-f_{\alpha} f_{\beta^{*}}\right) B_{\alpha \beta}(\boldsymbol{N} \cdot \boldsymbol{V},|\boldsymbol{V}|) d \boldsymbol{\xi}_{*} d \boldsymbol{N}$

where the Greek letters $\alpha$ and $\beta$ will be used symbolically to represent the gas species, i.e., $\{\alpha, \beta\}=\{A, B\} ; f_{\alpha} \equiv f_{\alpha}(\boldsymbol{x}, \boldsymbol{\xi}, t)$ represents the distribution function of species $\alpha$ with particle velocity $\boldsymbol{\xi}$ at position $\boldsymbol{x}$ and time $t$ in 3 -dimensional physical space; $Q_{\alpha}(f, f)$ is the Boltzmann collision operator for species $\alpha, B_{\alpha \beta}(\boldsymbol{N} \cdot \boldsymbol{V},|\boldsymbol{V}|)$ is the collision kernel which is decided by the intermolecular force between species $\alpha$ and $\beta, \boldsymbol{\xi}$ and $\boldsymbol{\xi}_{*}$ are pre-collision velocities, $\boldsymbol{N}$ is a unit vector and $B_{+}$is the semi-sphere defined by $\boldsymbol{N} \cdot \boldsymbol{V}=0$, where $\boldsymbol{V}$ is the relative velocity

$$
\boldsymbol{V}=\boldsymbol{\xi}-\boldsymbol{\xi}_{*}
$$

From conservation laws of momentum and energy:

$$
\left\{\begin{array}{l}
m_{\alpha} \boldsymbol{\xi}+m_{\beta} \boldsymbol{\xi}_{*}=m_{\alpha} \boldsymbol{\xi}^{\prime}+m_{\beta} \boldsymbol{\xi}_{*}^{\prime}, \\
m_{\alpha}|\xi|^{2}+m_{\beta}\left|\xi_{*}\right|^{2}=m_{\alpha}\left|\xi^{\prime}\right|^{2}+\left.m_{\mid} \xi_{*}^{\prime}\right|^{2},
\end{array}\right.
$$

the post-collision velocities $\boldsymbol{\xi}^{\prime}$ and $\boldsymbol{\xi}_{*}^{\prime}$ can be written as

$$
\left\{\begin{array}{l}
\boldsymbol{\xi}^{\prime}=\boldsymbol{\xi}-\frac{2 m_{\alpha \beta}}{m_{\alpha}} \boldsymbol{N}\left[\left(\boldsymbol{\xi}-\boldsymbol{\xi}_{*}\right) \cdot \boldsymbol{N}\right], \\
\boldsymbol{\xi}_{*}^{\prime}=\boldsymbol{\xi}_{*}+\frac{2 m_{\alpha \beta}}{m_{\beta}} \boldsymbol{N}\left[\left(\boldsymbol{\xi}-\boldsymbol{\xi}_{*}\right) \cdot \boldsymbol{N}\right],
\end{array}\right.
$$


with the reduced mass being

$$
m_{\alpha \beta}=\frac{m_{\alpha} m_{\beta}}{\left(m_{\alpha}+m_{\beta}\right)},
$$

in which $m_{\alpha}$ and $m_{\beta}$ are the molecular masses of species $\alpha$ and $\beta$, respectively. Without loss of generality, we assume $m_{A}<m_{B}$.

Furthermore, the macroscopic quantities of species $\alpha$, such as the molecular number density $n_{\alpha}$, mass density $\rho_{\alpha}$, flow velocity $\boldsymbol{u}_{\alpha}$, the total energy $E_{\alpha}$, and the internal energy $\epsilon_{\alpha}$ are calculated as the moments of distribution function $f_{\alpha}$ :

$$
\begin{aligned}
\rho_{\alpha} & =\int f_{\alpha} d \boldsymbol{\xi}, \quad n_{\alpha}=\rho_{\alpha} / m_{\alpha}, \\
\rho_{\alpha} \boldsymbol{u}_{\alpha} & =\int \boldsymbol{\xi} f_{\alpha} d \boldsymbol{\xi} \\
\rho_{\alpha} E_{\alpha} & =\frac{1}{2} \int \xi^{2} f_{\alpha} d \boldsymbol{\xi}=\frac{1}{2} \rho_{\alpha} u_{\alpha}^{2}+\epsilon_{\alpha}, \\
\epsilon_{\alpha} & =\frac{1}{2} \int\left|\boldsymbol{c}_{\alpha}\right|^{2} f_{\alpha} d \boldsymbol{\xi},
\end{aligned}
$$

where $\boldsymbol{c}_{\alpha}=\boldsymbol{\xi}-\boldsymbol{u}_{\alpha}$. The mass density $\rho_{m}$, number density $n_{m}$, flow velocity $\boldsymbol{u}_{m}$, energy $E_{m}$ and internal energy $\epsilon_{m}$ of the mixture can then be obtained as

$$
\begin{aligned}
\rho_{m} & =\sum_{\alpha=A, B} \rho_{\alpha}, \quad n_{m}=\sum_{\alpha=A, B} n_{\alpha}, \\
\rho_{m} \boldsymbol{u}_{m} & =\sum_{\alpha=A, B} \rho_{\alpha} \boldsymbol{u}_{\alpha}, \\
\rho_{m} E_{m} & =\sum_{\alpha=A, B} \rho_{\alpha} E_{\alpha}=\frac{1}{2} \rho_{m}\left|u_{m}\right|^{2}+\epsilon_{m} .
\end{aligned}
$$

The AAP model is a relaxation approximation of the full Boltzmann equation in Eq. (1)

$$
\frac{\partial f_{\alpha}}{\partial t}+\boldsymbol{\xi} \cdot \nabla f_{\alpha}=\Omega_{\alpha}(f, f)=\frac{f_{\alpha}^{*}-f_{\alpha}}{\tau_{\alpha}}
$$

where $\alpha=A$ or $B$ with

$$
f_{\alpha}^{*}=\rho_{\alpha}\left(\frac{m_{\alpha}}{2 \pi k_{B} T_{\alpha}^{*}}\right)^{\frac{3}{2}} \exp \left[-\frac{m_{\alpha}}{2 k_{B} T_{\alpha}^{*}}\left(\boldsymbol{\xi}-\boldsymbol{u}_{\alpha}^{*}\right)^{2}\right]
$$

in which $k_{B}$ is the Boltzmann constant. The parameters $\boldsymbol{u}_{\alpha}^{*}$ and $T_{\alpha}^{*}$ are introduced to recover the correct inter-species transfer of momentum and energy due to the collisions 
between molecules of different species [16],

$$
\begin{aligned}
\boldsymbol{u}_{\alpha}^{*} & =\boldsymbol{u}_{\alpha}+\tau_{\alpha} \sum_{\beta=A, B} 2 \frac{\rho_{\beta}}{m_{\alpha}+m_{\beta}} \theta_{\alpha \beta}\left(\boldsymbol{u}_{\beta}-\boldsymbol{u}_{\alpha}\right) \\
\frac{3}{2} k_{B} T_{\alpha}^{*} & =\frac{3}{2} k_{B} T_{\alpha}-\frac{m_{\alpha}}{2}\left(\boldsymbol{u}_{\alpha}^{*}-\boldsymbol{u}_{\alpha}\right)^{2} \\
& +\tau_{\alpha} \sum_{\beta=A, B} 4 m_{\alpha} \frac{\rho_{\beta}}{\left(m_{\alpha}+m_{\beta}\right)^{2}} \theta_{\alpha \beta}\left[\frac{3}{2} k_{B} T_{\beta}-\frac{3}{2} k_{B} T_{\alpha}+\frac{m_{\beta}}{2}\left(\boldsymbol{u}_{\beta}-\boldsymbol{u}_{\alpha}\right)^{2}\right],
\end{aligned}
$$

with $\theta_{\alpha \beta}$ being the interaction coefficient between molecules. The mean collision time and frequency from the AAP model can be expressed as

$$
\nu_{\alpha}=\frac{1}{\tau_{\alpha}}=\chi \sum_{\beta=A, B} \frac{\theta_{\alpha \beta} \rho_{\beta}}{m_{\beta}},
$$

where $\chi$ is either 1 or selected to make $\tau_{\alpha}$ be the same with that of the single-species gas when all species are equal. In this paper, we fix $\chi=1$. The interaction coefficient $\theta_{\alpha \beta}$ is related to the molecular interaction model. For example, for Maxwell molecules [44]:

$$
\theta_{\alpha \beta}=0.422 \pi\left[\frac{a_{\alpha \beta}\left(m_{\alpha}+m_{\beta}\right)}{m_{\alpha} m_{\beta}}\right]^{\frac{1}{2}},
$$

where $a_{\alpha \beta}$ is the constant of proportionality, i.e.,

$$
U_{\alpha \beta}=\frac{a_{\alpha \beta}}{4 r^{4}}
$$

where $U_{\alpha \beta}$ is the potential between two molecules of masses $m_{\alpha}$ and $m_{\beta}$ with a distance $r$.

In this work, we will consider Maxwell molecules and the force constant ratios $a_{B B} / a_{A A}$ and $a_{A B} / a_{A A}$ should be known. According to the viscosity of the mixture given by the AAP model [16]

$$
\mu=k_{B} T_{0} \sum_{\alpha=A, B} \frac{n_{\alpha}}{\nu_{\alpha}},
$$

if we take $n_{\alpha}=0$ then the binary gas mixture reduces to a single gas of species $\beta$ and its viscosity is

$$
\mu_{\beta}=k_{B} T_{0} \frac{n_{\beta}}{\nu_{\beta}}=k_{B} T_{0} \frac{1}{\theta_{\beta \beta}} .
$$

Then, based on Eq. (13), we can obtain

$$
\frac{a_{\beta \beta}}{a_{\alpha \alpha}}=\left(\frac{\mu_{\alpha}}{\mu_{\beta}}\right)^{2} \frac{m_{\beta}}{m_{\alpha}} .
$$

The constant $a_{\alpha \beta}$ can then be determined as [13]

$$
a_{\alpha \beta}=\sqrt{a_{\alpha \alpha} a_{\beta \beta}} .
$$




\section{DISCRETE UNIFIED GAS KINETIC SCHEME}

\section{A. Updating of the cell-averaged distribution function}

For problems of $D<3$ dimensional, the kinetic equation (9) can be simplified by introducing a reduced one. Specially, the original distribution function $f_{\alpha}$ can be expressed as $f_{\alpha}=f_{\alpha}(\boldsymbol{x}, \boldsymbol{\xi}, \boldsymbol{\eta}, t)$, where $\boldsymbol{\xi}=\left(\xi_{1}, \ldots, \xi_{D}\right), \boldsymbol{x}=\left(x_{1}, \ldots, x_{D}\right)$ and $\boldsymbol{\eta}=\left(\xi_{D+1}, \ldots, \xi_{3}\right)$ is a vector of length $L=3-D$, consisting of the rest components of the three-dimensional (3D) velocity space $\left(\xi_{1}, \xi_{2}, \xi_{3}\right)$. Since the evolution of the distribution function $f_{\alpha}$ is only relevant to the $D$-dimensional velocity and independent of $\boldsymbol{\eta}$, a reduced distribution function is used to remove the dependence of the redundant variable $\boldsymbol{\eta}[45,46]$

$$
g_{\alpha}(\boldsymbol{x}, \boldsymbol{\xi}, t)=\int f_{\alpha}(\boldsymbol{x}, \boldsymbol{\xi}, \boldsymbol{\eta}, t) d \boldsymbol{\eta} .
$$

However, the energy defined by Eq. (7) cannot be determined by this $g_{\alpha}$ solely, and another reduced distribution function is required,

$$
h_{\alpha}(\boldsymbol{x}, \boldsymbol{\xi}, t)=\int \eta^{2} f_{\alpha}(\boldsymbol{x}, \boldsymbol{\xi}, \boldsymbol{\eta}, t) d \boldsymbol{\eta} .
$$

The macroscopic flow variables of species $\alpha$ can be computed from the two reduced distribution functions as

$$
\begin{aligned}
& \rho_{\alpha}=\int g_{\alpha} d \boldsymbol{\xi}, \quad \rho_{\alpha} \boldsymbol{u}_{\alpha}=\int \boldsymbol{\xi} g_{\alpha} d \boldsymbol{\xi}, \\
& \rho_{\alpha} E_{\alpha}=\frac{1}{2} \int\left(\xi^{2} g_{\alpha}+h_{\alpha}\right) d \boldsymbol{\xi}
\end{aligned}
$$

and the heat flux $\boldsymbol{q}_{\alpha}$ and stress tensor $\boldsymbol{P}_{\alpha}$ can be computed as

$$
\boldsymbol{q}_{\alpha}=\frac{1}{2} \int \boldsymbol{c}_{\alpha}\left(c_{\alpha}^{2} g_{\alpha}+h_{\alpha}\right) d \boldsymbol{\xi}, \quad \boldsymbol{P}_{\alpha}=\int \boldsymbol{c}_{\alpha} \boldsymbol{c}_{\alpha}\left(g_{\alpha}-g_{\alpha}^{e q}\right) d \boldsymbol{\xi},
$$

where

$$
g_{\alpha}^{e q}=\rho_{\alpha}\left(\frac{m_{\alpha}}{2 \pi k_{B} T_{\alpha}}\right)^{D / 2} \exp \left[-\frac{m_{\alpha}}{2 k_{B} T_{\alpha}}\left(\boldsymbol{\xi}-\boldsymbol{u}_{\alpha}\right)^{2}\right] .
$$

Note that $g_{\alpha}^{e q}$ cannot give any communication among species in the multispecies system.

The evolution equations for $g_{\alpha}$ and $h_{\alpha}$ can be deduced from Eq. (9)

$$
\begin{gathered}
\frac{\partial g_{\alpha}}{\partial t}+\boldsymbol{\xi} \cdot \nabla g_{\alpha}=\Omega\left(g_{\alpha}\right) \equiv \frac{g_{\alpha}^{*}-g_{\alpha}}{\tau_{\alpha}}, \\
\frac{\partial h_{\alpha}}{\partial t}+\boldsymbol{\xi} \cdot \nabla h_{\alpha}=\Omega\left(h_{\alpha}\right) \equiv \frac{h_{\alpha}^{*}-h_{\alpha}}{\tau_{\alpha}},
\end{gathered}
$$


where

$$
\begin{aligned}
g_{\alpha}^{*} & =\rho_{\alpha}\left(\frac{m_{\alpha}}{2 \pi k_{B} T_{\alpha}^{*}}\right)^{D / 2} \exp \left[-\frac{m_{\alpha}}{2 k_{B} T_{\alpha}^{*}}\left(\boldsymbol{\xi}-\boldsymbol{u}_{\alpha}^{*}\right)^{2}\right], \\
h_{\alpha}^{*} & =(3-D) R_{\alpha} T_{\alpha}^{*} g_{\alpha}^{*},
\end{aligned}
$$

with $R_{\alpha}=k_{B} / m_{\alpha}$.

Then the DUGKS for a binary gas mixture is constructed based on Eq. (24), which can be rewritten as

$$
\frac{\partial \phi_{\alpha}}{\partial t}+\boldsymbol{\xi} \cdot \nabla \phi_{\alpha}=\Omega_{\alpha} \equiv \frac{\phi_{\alpha}^{*}-\phi_{\alpha}}{\tau_{\alpha}}
$$

where $\phi_{\alpha}=g_{\alpha}$ or $h_{\alpha}, \phi_{\alpha}^{*}=g_{\alpha}^{*}$ or $h_{\alpha}^{*}$. As a finite volume scheme, the computation domain is first divided into a set of control volumes (cells). Then integrating Eq. (26) over a control volume $V_{j}$ centered at $\boldsymbol{x}_{j}$ from time $t_{n}$ to $t_{n+1}$, and employing the midpoint rule for the time integration of the convective term and trapezoidal rule for the collision term inside each cell, the evolution equation for species $\alpha$ can be written as

$$
\phi_{\alpha, j}^{n+1}(\boldsymbol{\xi})-\phi_{\alpha, j}^{n}(\boldsymbol{\xi})=-\frac{\Delta t}{\left|V_{j}\right|} \mathcal{F}_{\alpha, j}^{n+1 / 2}(\boldsymbol{\xi})+\frac{\Delta t}{2}\left[\Omega_{\alpha, j}^{n}(\boldsymbol{\xi})+\Omega_{\alpha, j}^{n+1}(\boldsymbol{\xi})\right],
$$

here $\Delta t=t_{n+1}-t_{n}$ is the time step, $\left|V_{j}\right|$ is the volume of the cell $V_{j}$ and $\phi_{\alpha, j}^{n}$ and $\Omega_{\alpha, j}^{n}$ are the cell averaged values of $\phi_{\alpha}$ and $\Omega_{\alpha}$ defined by

$$
\begin{aligned}
\phi_{\alpha, j}^{n}(\boldsymbol{\xi}) & =\frac{1}{\left|V_{j}\right|} \int_{V_{j}} \phi_{\alpha}\left(\boldsymbol{x}, \boldsymbol{\xi}, t_{n}\right) d \boldsymbol{x}, \\
\Omega_{\alpha, j}^{n}(\boldsymbol{\xi}) & =\frac{1}{\left|V_{j}\right|} \int_{V_{j}} \Omega_{\alpha}\left(\boldsymbol{x}, \boldsymbol{\xi}, t_{n}\right) d \boldsymbol{x} .
\end{aligned}
$$

The term $\mathcal{F}_{\alpha, j}^{n+1 / 2}$ in Eq. (27) is the distribution function flux across the cell interface

$$
\mathcal{F}_{\alpha, j}^{n+1 / 2}(\boldsymbol{\xi})=\int_{\partial V_{j}}(\boldsymbol{\xi} \cdot \boldsymbol{n}) \phi_{\alpha}\left(\boldsymbol{x}, \boldsymbol{\xi}, t_{n+1 / 2}\right) d \boldsymbol{S},
$$

where $\boldsymbol{n}$ is the outward unit vector normal to the cell surface $\partial V_{j}$. It is clear that the updating rule given by Eq. (27) is implicit due to the term $\Omega_{\alpha, j}^{n+1}$. In order to obtain an explicit form, two new distribution functions are introduced

$$
\begin{aligned}
& \tilde{\phi}_{\alpha}=\phi_{\alpha}-\frac{\Delta t}{2} \Omega_{\alpha}=\frac{2 \tau_{\alpha}+\Delta t}{2 \tau_{\alpha}} \phi_{\alpha}-\frac{\Delta t}{2 \tau_{\alpha}} \phi_{\alpha}^{*}, \\
& \tilde{\phi}_{\alpha}^{+}=\phi_{\alpha}+\frac{\Delta t}{2} \Omega_{\alpha}=\frac{2 \tau_{\alpha}-\Delta t}{2 \tau_{\alpha}+\Delta t} \phi_{\alpha}+\frac{2 \Delta t}{2 \tau_{\alpha}+\Delta t} \phi_{\alpha}^{*} .
\end{aligned}
$$


Then Eq. (27) can be rewritten as

$$
\tilde{\phi}_{\alpha, j}^{n+1}=\tilde{\phi}_{\alpha, j}^{+, n}-\frac{\Delta t}{\left|V_{j}\right|} \mathcal{F}_{\alpha, j}^{n+1 / 2}
$$

To avoid the implicity of Eq. (27), we can track the evolution of $\tilde{\phi}_{\alpha}$ instead of the original distribution function $\phi_{\alpha}$.

Note that the moments of $\tilde{g}_{\alpha}$ and $\tilde{h}_{\alpha}$ have the following expressions:

$$
\begin{aligned}
\int \tilde{g}_{\alpha} d \boldsymbol{\xi} & =\rho_{\alpha}, \\
\int \boldsymbol{\xi} \tilde{g}_{\alpha} d \boldsymbol{\xi} & =\frac{2 \tau_{\alpha}+\Delta t}{2 \tau_{\alpha}} \int \boldsymbol{\xi} g_{\alpha} d \boldsymbol{\xi}-\frac{\Delta t}{2 \tau_{\alpha}} \int \boldsymbol{\xi} g_{\alpha}^{*} d \boldsymbol{\xi} \\
& =\frac{2 \tau_{\alpha}+\Delta t}{2 \tau_{\alpha}} \rho_{\alpha} \boldsymbol{u}_{\alpha}-\frac{\Delta t}{2 \tau_{\alpha}} \rho_{\alpha} \boldsymbol{u}_{\alpha}^{*}, \\
\frac{1}{2} \int\left(\xi^{2} \tilde{g}_{\alpha}+\tilde{h}_{\alpha}\right) d \boldsymbol{\xi} & =\frac{2 \tau_{\alpha}+\Delta t}{2 \tau_{\alpha}} \int \frac{1}{2}\left(\xi^{2} g_{\alpha}+h_{\alpha}\right) d \boldsymbol{\xi}-\frac{\Delta t}{2 \tau_{\alpha}} \int \frac{1}{2}\left(\xi^{2} g_{\alpha}^{*}+h_{\alpha}^{*}\right) d \boldsymbol{\xi} \\
& =\frac{2 \tau_{\alpha}+\Delta t}{2 \tau_{\alpha}} \rho_{\alpha} E_{\alpha}-\frac{\Delta t}{2 \tau_{\alpha}} \rho_{\alpha} E_{\alpha}^{*},
\end{aligned}
$$

where $\rho_{\alpha} E_{\alpha}^{*}=\rho_{\alpha} u_{\alpha}^{* 2} / 2+\rho_{\alpha} R_{\alpha} T_{\alpha}^{*} /(\gamma-1)$ with $\gamma$ being the the specific heat ratio. Once the distribution functions $\tilde{g}_{\alpha}$ and $\tilde{h}_{\alpha}$ are known, the macroscopic variables $\boldsymbol{W}_{\alpha}=$ $\left(\rho_{\alpha}, \rho_{\alpha} \boldsymbol{u}_{\alpha}, \rho_{\alpha} E_{\alpha}\right)$ and $\boldsymbol{W}_{\alpha}^{*}=\left(\rho_{\alpha}, \rho_{\alpha} \boldsymbol{u}_{\alpha}^{*}, \rho_{\alpha} E_{\alpha}^{*}\right)$ can be solved from the moments of them according to Eq. (32). Then Eq. (32) becomes an equation set with four relations [[Eq. (32b) and Eq. (32c) for $\alpha=A, B]$ and eight unknown variables $\left(\boldsymbol{u}_{\alpha}, T_{\alpha}, \boldsymbol{u}_{\alpha}^{*}\right.$ and $T_{\alpha}^{*}$ for $\left.\alpha=A, B\right)$. To ensure the equation set is closed, the exchange relations between species $A$ and $B$ in Eq. (11) are introduced. Then the macroscopic quantities $\boldsymbol{u}_{\alpha}, T_{\alpha}, \boldsymbol{u}_{\alpha}^{*}$ and $T_{\alpha}^{*}$ for each species can be solved analytically from Eqs. (32) and (11). In particular, for Maxwell molecules, the interaction coefficients $\theta_{\alpha \beta}$ and $\theta_{\alpha \alpha}$ only depend on the mass ratio [see Eq. (13)]. By this way, the macroscopic variables $\boldsymbol{W}_{\alpha}$ and $\boldsymbol{W}_{\alpha}^{*}$ for each species can be expressed in terms of the moments of $\tilde{g}_{\alpha}$ and $\tilde{h}_{\alpha}$ explicitly.

\section{B. Flux evaluation}

To update $\tilde{\phi}_{\alpha, j}^{n+1}$ according to Eq. (31), the flux $\mathcal{F}^{n+1 / 2}$ should be evaluated. Based on the definition of flux in Eq. (29), $\mathcal{F}^{n+1 / 2}$ can be calculated by reconstructing the distribution function $\phi_{\alpha}\left(\boldsymbol{x}, \boldsymbol{\xi}, t_{n+1 / 2}\right)$ at the cell interface. To this end, Eq. (26) is integrated along the characteristic line from time $t_{n}$ to $t_{n+1 / 2}$,

$$
\phi_{\alpha}\left(\boldsymbol{x}_{b}, \boldsymbol{\xi}, t_{n}+s\right)-\phi_{\alpha}\left(\boldsymbol{x}_{b}-\boldsymbol{\xi} s, \boldsymbol{\xi}, t_{n}\right)=\frac{s}{2}\left[\Omega_{\alpha}\left(\boldsymbol{x}_{b}, \boldsymbol{\xi}, t_{n}+s\right)+\Omega_{\alpha}\left(\boldsymbol{x}_{b}-\boldsymbol{\xi} s, \boldsymbol{\xi}, t_{n}\right)\right],
$$


where $s=\Delta t / 2$ and $\boldsymbol{x}_{b}$ is the interface center of cell $j$, and the trapezoidal rule is applied to evaluate the collision term again. In order to remove the implicity caused by the term $\Omega_{\alpha}^{n+1 / 2}$, another two auxiliary distribution functions are introduced,

$$
\begin{aligned}
& \bar{\phi}_{\alpha}=\phi_{\alpha}-\frac{s}{2} \Omega_{\alpha}=\frac{2 \tau_{\alpha}+s}{2 \tau_{\alpha}} \phi_{\alpha}-\frac{s}{2 \tau_{\alpha}} \phi_{\alpha}^{*}, \\
& \bar{\phi}_{\alpha}^{+}=\phi_{\alpha}+\frac{s}{2} \Omega_{\alpha}=\frac{2 \tau_{\alpha}-s}{2 \tau_{\alpha}+s} \phi_{\alpha}+\frac{2 s}{2 \tau_{\alpha}+s} \phi_{\alpha}^{*} .
\end{aligned}
$$

Then Eq. (33) can be rewritten as

$$
\bar{\phi}_{\alpha}\left(\boldsymbol{x}_{b}, \boldsymbol{\xi}, t_{n+1 / 2}\right)=\bar{\phi}_{\alpha}^{+}\left(\boldsymbol{x}_{b}-\boldsymbol{\xi} s, \boldsymbol{\xi}, t_{n}\right)
$$

where

$$
\bar{\phi}_{\alpha}^{+}\left(\boldsymbol{x}_{b}-\boldsymbol{\xi} s, \boldsymbol{\xi}, t_{n}\right)=\bar{\phi}_{\alpha}^{+}\left(\boldsymbol{x}_{j}, \boldsymbol{\xi}, t_{n}\right)+\left(\boldsymbol{x}_{b}-\boldsymbol{x}_{j}-\boldsymbol{\xi} s\right) \cdot \boldsymbol{\delta}_{j}, \quad\left(\boldsymbol{x}_{b}-\boldsymbol{\xi} s\right) \in V_{j} .
$$

Here $\boldsymbol{\delta}_{j}$ is the slope of $\bar{\phi}_{\alpha}^{+}$in cell $j$. For example, in the one-dimensional (1D) case, the distribution function $\phi_{\alpha}$ at the cell interface $x_{b}=x_{j+1 / 2}$ is reconstructed through approximating the distribution function $\bar{\phi}_{\alpha}^{+}$as

$$
\bar{\phi}_{\alpha}^{+}\left(x_{b}-\xi s, \xi, t_{n}\right)= \begin{cases}\bar{\phi}_{\alpha}^{+}\left(x_{j}, \xi, t_{n}\right)+\left(x_{b}-x_{j}-\xi s\right) \cdot \delta_{j}, & \xi>0, \\ \bar{\phi}_{\alpha}^{+}\left(x_{j+1}, \xi, t_{n}\right)+\left(x_{b}-x_{j+1}-\xi s\right) \cdot \delta_{j}, & \xi<0 .\end{cases}
$$

Here the van Leer limiter [47] is applied to determine the slope $\boldsymbol{\delta}_{j}$ for discontinuous problems. Once the distribution function $\bar{\phi}_{\alpha}$ at the interface is known, the original distribution function $\phi_{\alpha}$ can be obtained according to Eq. (34), i.e.,

$$
\phi_{\alpha}\left(\boldsymbol{x}_{b}, \boldsymbol{\xi}, t_{n+1 / 2}\right)=\frac{2 \tau_{\alpha}}{2 \tau_{\alpha}+s} \bar{\phi}_{\alpha}\left(\boldsymbol{x}_{b}, \boldsymbol{\xi}, t_{n}+s\right)+\frac{s}{2 \tau_{\alpha}+s} \phi_{\alpha}^{*}\left(\boldsymbol{x}_{b}, \boldsymbol{\xi}, t_{n}+s\right) .
$$

Note that the macroscopic variables used to evaluate the distribution function $\phi_{\alpha}^{*}$ are computed from $\bar{\phi}_{\alpha}$ directly as

$$
\begin{aligned}
\int \bar{g}_{\alpha} d \boldsymbol{\xi} & =\rho_{\alpha} \\
\int \boldsymbol{\xi} \bar{g}_{\alpha} d \boldsymbol{\xi} & =\frac{2 \tau_{\alpha}+s}{2 \tau_{\alpha}} \int \boldsymbol{\xi} g_{\alpha} d \boldsymbol{\xi}-\frac{s}{2 \tau_{\alpha}} \int \boldsymbol{\xi} g_{\alpha}^{*} d \boldsymbol{\xi} \\
& =\frac{2 \tau_{\alpha}+s}{2 \tau_{\alpha}} \rho_{\alpha} \boldsymbol{u}_{\alpha}-\frac{s}{2 \tau_{\alpha}} \rho_{\alpha} \boldsymbol{u}_{\alpha}^{*}, \\
\frac{1}{2} \int\left(\xi^{2} \bar{g}_{\alpha}+\bar{h}_{\alpha}\right) d \boldsymbol{\xi} & =\frac{2 \tau_{\alpha}+s}{2 \tau_{\alpha}} \int \frac{1}{2}\left(\xi^{2} g_{\alpha}+h_{\alpha}\right) d \boldsymbol{\xi}-\frac{s}{2 \tau_{\alpha}} \int \frac{1}{2}\left(\xi^{2} g_{\alpha}^{*}+h_{\alpha}^{*}\right) d \boldsymbol{\xi} \\
& =\frac{2 \tau_{\alpha}+s}{2 \tau_{\alpha}} \rho_{\alpha} E_{\alpha}-\frac{s}{2 \tau_{\alpha}} \rho_{\alpha} E_{\alpha}^{*} .
\end{aligned}
$$


Similar to the previous treatment of cell averaged macroscopic variables, equations (39) and (11) constitute a closed system for eight unknown variables $\left(\boldsymbol{u}_{\alpha}, T_{\alpha}, \boldsymbol{u}_{\alpha}^{*}\right.$ and $T_{\alpha}^{*}$ at cell interface $\boldsymbol{x}_{b}$ and half time step $t_{n+1 / 2}$ for each species $\alpha$ ). These macroscopic variables can also be obtained explicitly, and the original distribution function $\phi_{\alpha}$ can be updated from Eq. (38). Then the flux across each cell interface can be computed according to Eq. (29). Finally, the cell averaged distribution function $\tilde{\phi}_{\alpha}$ in each cell is updated from $t_{n}$ to $t_{n+1}$ according to Eq. (31).

In practical implementation, the velocity space is discretized into a set of discrete velocities $\boldsymbol{\xi}_{i}(i=1,2, \ldots, b)$. Certain quadrature rules, such as the Gaussian-Hermite or NewtonCotes formula, can be chosen to discretize the velocity space and approximate the moments, e.g.,

$$
\begin{aligned}
& \rho_{\alpha}=\sum_{i} w_{i} \tilde{g}_{\alpha}\left(\boldsymbol{\xi}_{i}\right), \quad \rho_{\alpha} \boldsymbol{u}_{\alpha}=\sum_{i} w_{i} \boldsymbol{\xi}_{i} \tilde{g}_{\alpha}\left(\boldsymbol{\xi}_{i}\right), \\
& \rho_{\alpha} E_{\alpha}=\frac{1}{2} \sum_{i} w_{i}\left(\xi_{i}^{2} \tilde{g}_{\alpha}\left(\boldsymbol{\xi}_{i}\right)+\tilde{h}_{\alpha}\left(\boldsymbol{\xi}_{i}\right)\right),
\end{aligned}
$$

where $w_{i}$ are the quadrature weights.

The time step in DUGKS is determined by the Courant-Friedrichs-Lewy(CFL) condition,

$$
\Delta t=\varsigma \frac{\Delta x}{U_{m}+\xi_{m}},
$$

where $0<\varsigma<1$ is the CFL number, $\Delta x$ is the minimal mesh size, $\xi_{m}$ is the maximum discrete velocity, and $U_{m}$ is the maximum flow velocity. Due to the coupling of particle transport and collision in the reconstruction of the interface distribution function, the DUGKS has the asymptotic preserving (AP) property [30, 32]. As a result, the time step $\Delta t$ is not limited by the particle collision time but determined by the CFL number, and the DUGKS is uniformly stable with respect to the Knudsen number.

\section{Algorithm}

Now we list the computational procedure of the DUGKS for a binary gas mixture made up of Maxwell molecules from $t_{n}$ to $t_{n+1}$ :

1. Calculate $\tilde{\phi}_{\alpha}^{+, n}$ and $\bar{\phi}_{\alpha}^{+, n}$ at each cell center according to Eqs. (30) and (34), respectively.

2. Reconstruct the distribution function $\bar{\phi}_{\alpha}^{+}\left(\boldsymbol{x}_{b}-\boldsymbol{\xi} s, \boldsymbol{\xi}, t_{n}\right)$ according to Eq. (36). 
3. Calculate the distribution function $\bar{\phi}_{\alpha}\left(\boldsymbol{x}_{b}, \boldsymbol{\xi}, t_{n+1 / 2}\right)$ according to Eq. (35).

4. Calculate the macroscopic variables $\boldsymbol{W}_{\alpha}\left(\boldsymbol{x}_{b}, t_{n+1 / 2}\right)$ and $\boldsymbol{W}_{\alpha}^{*}\left(\boldsymbol{x}_{b}, t_{n+1 / 2}\right)$ according to Eqs. (39) and (11).

5. Calculate the original distribution function at each cell interface $\phi_{\alpha}\left(\boldsymbol{x}_{b}, \boldsymbol{\xi}, t_{n+1 / 2}\right)$ according to Eq. (38).

6. Calculate the microflux $\mathcal{F}^{n+1 / 2}$ across each cell interface from $\phi_{\alpha}\left(\boldsymbol{x}_{b}, \boldsymbol{\xi}, t_{n+1 / 2}\right)$ according to Eq. (29).

7. Update the cell-averaged distribution function $\tilde{\phi}_{\alpha}$ in each cell according to Eq. (31).

\section{NUMERICAL EXAMPLES}

In this section, the proposed DUGKS will be validated by several test cases, including the shock structure problem under different Mach numbers, the channel flow driven by small pressure gradient, or temperature gradient or concentration gradient, and the $1 D$ and $2 D$ shear driven flows over a wide range of Knudsen numbers. In each test different mass ratios and molar concentrations will be considered.

\section{A. Shock structure}

The first test case is the shock structure for a binary gas mixture. Consider a normal shock formed by a mixture made up of a light species $(A)$ and a heavy species $(B)$. The molar concentrations, number densities, velocities and temperatures are expressed as $\chi_{1}^{A, B}$, $n_{1}^{A, B}, U_{1}, T_{1}$ in the upstream and $\chi_{2}^{A, B}, n_{1}^{A, B}, U_{2}, T_{2}$ in the downstream, where $\chi^{A, B}=$ $n^{A, B} /\left(n^{A}+n^{B}\right)$. The Mach number is defined as

$$
M a_{1}=\frac{U_{1}}{\left(\gamma k_{B} T_{1} / m\right)^{1 / 2}}
$$


where $m=m_{A} \chi^{A}+m_{B} \chi^{B}$. The Rankine-Hugoniot condition [48] holds for each species, so the downstream quantities are then determined as

$$
\begin{aligned}
& M a_{2}=\sqrt{\frac{M a_{1}^{2}(\gamma-1)+2}{2 \gamma M a_{1}^{2}-(\gamma-1)}}, \\
& \chi_{2}^{A, B}=\chi_{1}^{A, B}, \\
& \frac{n_{2}^{A}}{n_{1}^{A}}=\frac{n_{2}^{B}}{n_{1}^{B}}=\frac{(\gamma+1) M a_{1}^{2}}{(\gamma-1) M a_{1}^{2}+2}, \\
& \frac{T_{2}}{T_{1}}=\frac{\left[2+(\gamma-1) M a_{1}^{2}\right]\left(2 \gamma M a_{1}^{2}-\gamma+1\right)}{M a_{1}^{2}(\gamma+1)^{2}} .
\end{aligned}
$$

The reference mean free path is defined as [49]

$$
\lambda_{\infty}=\frac{\mu_{B}}{P_{0}} \sqrt{\frac{2 k_{B} T_{1}}{m_{B}}},
$$

where $P_{0}=n_{1} k_{B} T_{1}$ and $\mu_{B}$ is the viscosity of species $B$ defined in Eq. (16). In the simulations, the computation domain is set to be $\left[-25 \lambda_{\infty}, 25 \lambda_{\infty}\right]$, which is divided into 100 uniform cells. The velocity space is discretized by Newton-Cotes quadrature with 101 velocity points distributed uniformly in $\left[-8 \sqrt{2 k_{B} T_{1} / m}, 8 \sqrt{2 k_{B} T_{1} / m}\right]$. The CFL number is set to be 0.6 . The origin of the distribution is determined so that $n(0)=\left(n_{1}+n_{2}\right) / 2$. The simulation results are normalized as

$$
\widehat{n}^{A, B}=\frac{n^{A, B}-n_{1}^{A, B}}{n_{2}^{A, B}-n_{1}^{A, B}}, \quad \widehat{T}^{A, B}=\frac{T^{A, B}-T_{1}^{A, B}}{T_{2}^{A, B}-T_{1}^{A, B}},
$$

where the hat will be dropped for simplicity in the following.

The number density and temperature of each species under different Mach numbers and concentrations are shown and compared with those of the UGKS method [28, 29] in Figs. 13. Good agreement between the two methods can be observed. As shown in Fig. 1 for $M a_{1}=1.5$ and $m_{A} / m_{B}=0.5$, the number density $n_{A}$ and temperature $T_{A}$ of the light species firstly deviate from their upstream quantities, and $n_{A}$ reaches its downstream quantity earlier than $n_{B}$. However, the temperature of the heavy species, $T_{B}$, arises rapidly, then exceeds that of the light one $\left(T_{A}\right)$ and finally reaches its downstream quantity earlier. These phenomena become more obvious as the concentration of the light species becomes large. Figure 2 presents the results for smaller value of $m_{A} / m_{B}$ under $M a_{1}=1.5$. It can observed that the above features appear more clearly. The results with $M a_{1}=3$ and $m_{A} / m_{B}=0.5$ 
are shown in Fig. 3, and the above phenomena still exist. However, $T_{B}$ does not arise monotonically for large concentration of the light species. At the beginning, it approximates the maximum value and then decreases to its downstream quantity, which has been known as the temperature overshoot [50].

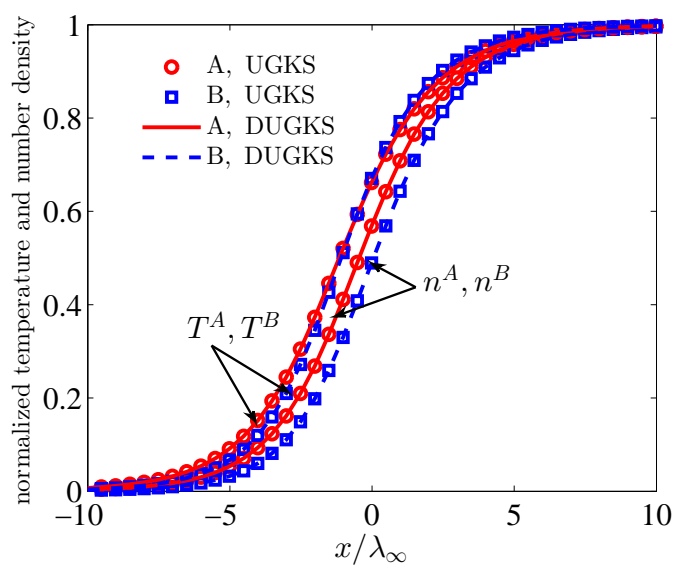

(a)

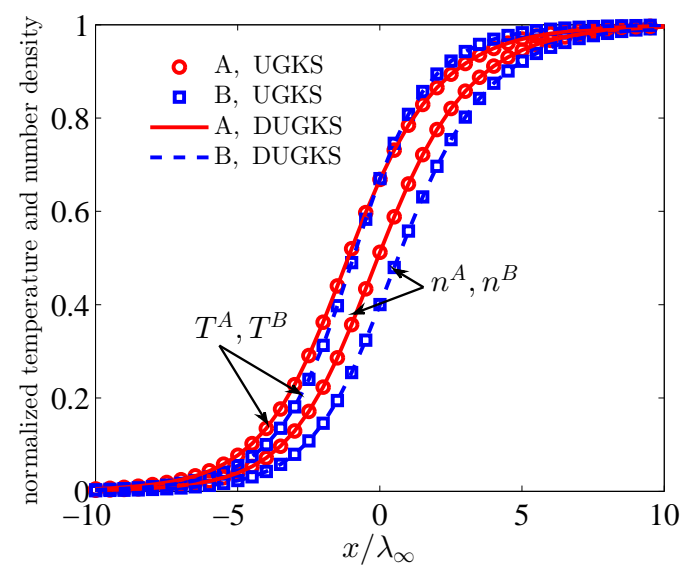

(b)

FIG. 1. Structure of a $M a_{1}=1.5$ shock wave in a binary gas mixture with $m_{A} / m_{B}=0.5$ and $a_{B B} / a_{A A}=1:$ (a) $\chi_{1}^{A}=0.1 ;$ (b) $\chi_{1}^{A}=0.9$.

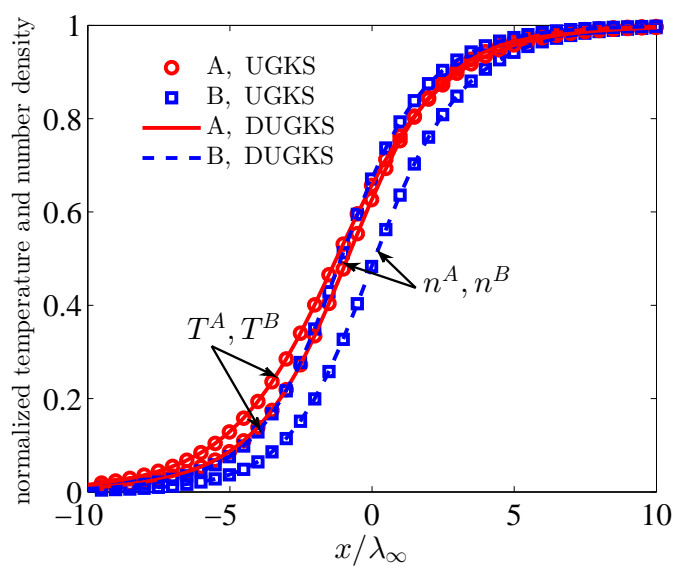

(a)

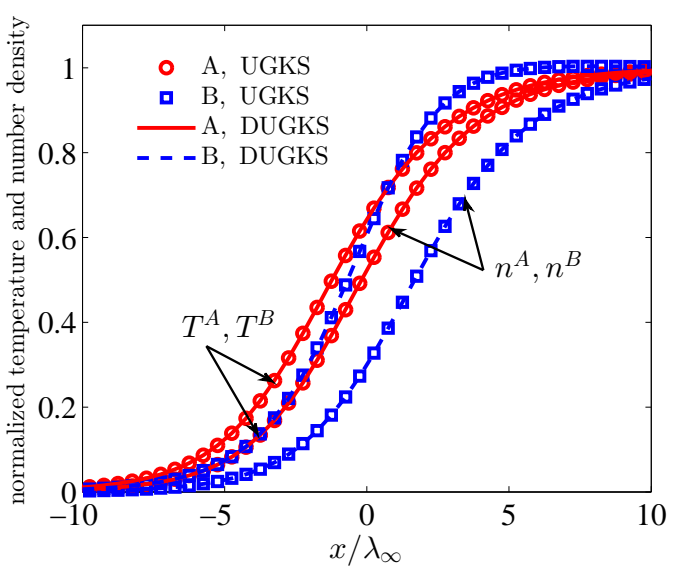

(b)

FIG. 2. Structure of a $M a_{1}=1.5$ shock wave in a binary gas mixture with $m_{A} / m_{B}=0.25$ and $a_{B B} / a_{A A}=1:$ (a) $\chi_{1}^{A}=0.1 ;$ (b) $\chi_{1}^{A}=0.9$. 


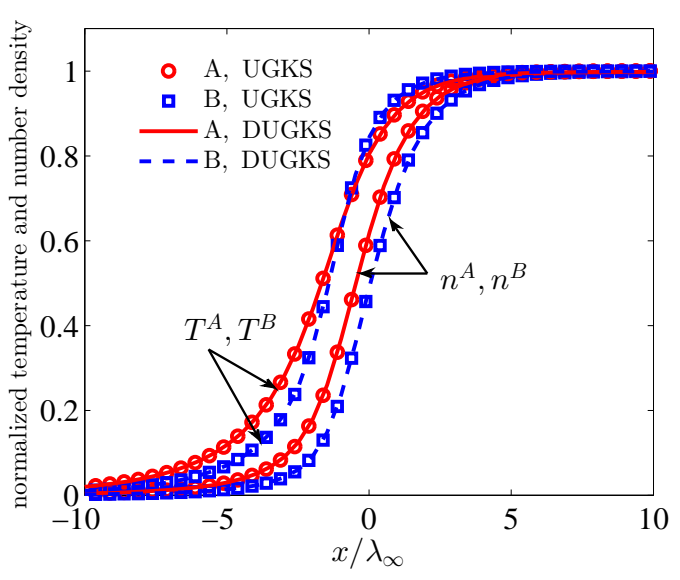

(a)

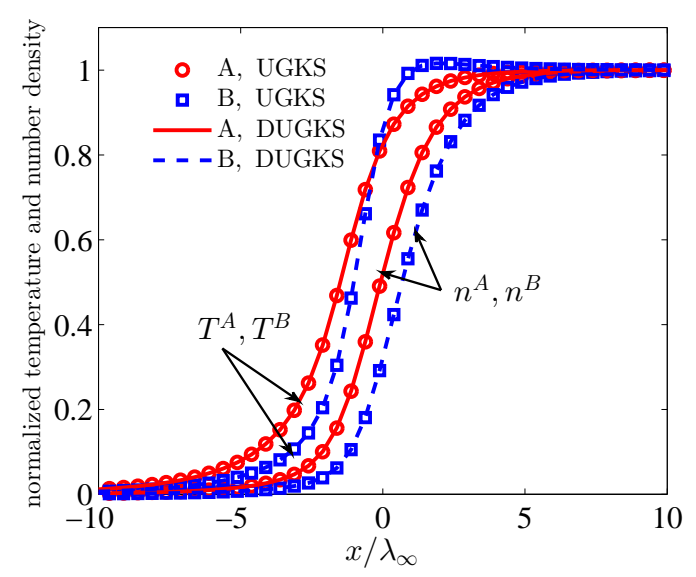

(b)

FIG. 3. Structure of a $M a_{1}=3.0$ shock wave in a binary gas mixture with $m_{A} / m_{B}=0.5$ and $a_{B B} / a_{A A}=1:$ (a) $\chi_{1}^{A}=0.1$; (b) $\chi_{1}^{A}=0.9$.

\section{B. Channel flow driven by a gradient}

Now we consider a binary mixture flow in a long channel with width $H$ (see Fig. 4). A uniform pressure gradient, or temperature gradient or concentration gradient exists along the channel ( $x$-direction), i.e., $p=p_{0}\left(1+C_{p} x / H\right), T=T_{0}\left(1+C_{T} x / H\right), \chi^{A}=\chi_{0}^{A}+C_{\chi} x / H$, where $C_{p}, C_{\chi}, C_{T}, p_{0}$ and $T_{0}$ are constants. Both plates are fully diffusive and a uniform temperature gradient $T_{0}\left(1+C_{T} x / H\right)$ maintains. The inlet and outlet are imposed with pressure boundary conditions based on characteristics as described in Ref. [28]. In this case,
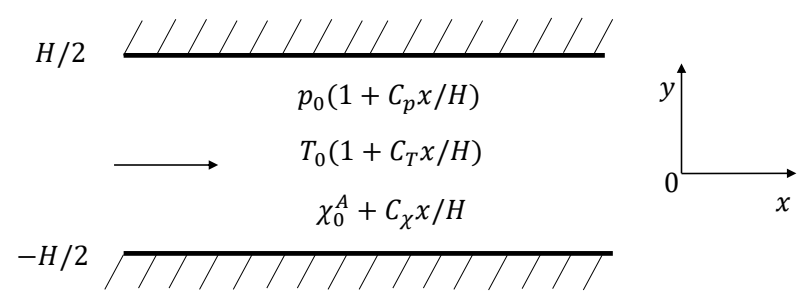

FIG. 4. Schematic of the channel flow.

the numerical results are compared with those from Kosuge [51] based on the McCormack model. The definitions of the intermolecular potential and the reference diameter for Maxwell molecules are [51]

$$
U_{\alpha \beta}=\frac{\kappa_{\alpha \beta}}{r^{4}}, \quad d_{*}^{\alpha \beta}=\left(\frac{4 \kappa_{\alpha \beta}}{2 k_{B} T_{0}}\right)^{\frac{1}{4}}
$$


in which $\kappa_{\alpha \beta}$ is a positive constant. Comparing Eq. (46) with Eq. (14), one can obtain that

$$
a_{\alpha \beta}=4 \kappa_{\alpha \beta}
$$

thus the relationship between $d_{*}^{\alpha \beta}$ and $a_{\alpha \beta}$ is

$$
d_{*}^{\alpha \beta}=\left(\frac{a_{\alpha \beta}}{2 k_{B} T_{0}}\right)^{\frac{1}{4}} .
$$

The reference mean free path is given by [51]

$$
\lambda=\frac{1}{\sqrt{2} \pi n_{0}\left(d_{*}^{A A}\right)^{2}},
$$

where $n_{0}$ is the total number density at the inlet. In the simulations, we consider the flow driven by the pressure gradient, temperature gradient, and concentration gradient separately. For the pressure driven flow, $C_{T}$ and $C_{\chi}$ are zero and the non-dimensional particle flux of each species is defined by [51]

$$
M_{p}^{A, B}=\frac{1}{C_{p}} \int_{-H / 2}^{H / 2} \frac{u_{A, B}}{\sqrt{2 k_{B} T_{0} / m_{A}}} d(y) .
$$

The fluxes $M_{T}^{A, B}$ and $M_{\chi}^{A, B}$ due to the temperature gradient and concentration gradient can be defined similarly.

In our simulations, the length-to-height ratio of the channel is set to be 40 and the gradient $C_{p}, C_{\chi}$ and $C_{T}$ are kept at 0.01 . The particle fluxes versus Knudsen number are displayed in Figs. 5-7 for $m_{B} / m_{A}=2,4$ and 10 with $a_{B B} / a_{A A}=a_{A B} / a_{A A}=1$. The results of the UGKS for binary gas mixtures [28, 29] of Maxwell molecules are also included for comparison. Overall good agreement between the DUGKS and UGKS can be observed for the cases considered.

In Fig. 5, the mass fluxes driven by a pressure gradient show good agreement with the solutions of the linearized Boltzmann equation [51], with some minor deviations. The differences can be attributed to the discrepancy in viscosity from different models. Furthermore, it can be observed that a minimum appears for each species around $\mathrm{Kn} \approx 1$, which is the well-known Knudsen minimum [52] for Poiseuille flow in rarefied regime.

Figure 6 shows the mass fluxes of each species for flows driven by a temperature gradient. It can be seen that $M_{T}^{A}$ and $M_{T}^{B}$ increase monotonically with $\mathrm{Kn}$, and their difference decreases with Kn. Furthermore, it is observed that the predicted mass fluxes of the heavy 
species $B$ match quite well with the reference solutions. But clear deviations can be observed for the light species $A$ and the deviations increase with Kn. This can be attributed to the different thermal conductivities from the AAP model and the linearized Boltzmann equation [51].

The mass fluxes at different Knudsen numbers driven by a concentration gradient are shown in Fig. 7. It can be seen that the concentration of the light species $A$ increases while that of the heavy one decreases along the channel. As a result, the light one turns to flow in the opposite direction. But it can be observed that both $\left|M_{\chi}^{A}\right|$ and $M_{\chi}^{B}$ grow monotonically with increasing $\mathrm{Kn}$, and the results predicted by the AAP model are in good agreement with the reference data for all cases considered.

It can also be observed that the differences in the mass fluxes of species $A$ and $B$ decrease with increasing Kn driven by the pressure, temperature, or concentration gradient. This is because the velocities of the two species are different due to the difference in molecular masses. Particularly, the differences between $u_{A}$ and $u_{B}$ can be much larger as the intermolecular collisions are insufficient such that the momentum of the two species are not exchanged sufficiently. On the contrary, the velocities of the two species will become closer in the continuum limit when the collision is frequent.

\section{Couette flow}

Now we consider the plane Couette flow of a binary gas mixture in the rarefied regime. As shown in Fig. 8, two plates with a constant temperature $T_{0}$ are fixed at $y= \pm H / 2$ and move with velocities $\pm U / 2$ in $x$-direction, respectively. The plates are fully diffusive and the periodic boundary conditions are imposed on the inlet and outlet of the channel. Here we assume that the plate velocities are much smaller than the characteristic molecular velocity $v_{0}$ of mixture, i.e.,

$$
U \ll v_{0}, \quad v_{0}=\sqrt{\frac{2 k_{B} T_{0}}{m}},
$$

where $m$ is the mean molecular mass of the mixture $m=C_{0} m_{A}+\left(1-C_{0}\right) m_{B}$, and $C_{0}$ is the molar concentration of the light species in equilibrium

$$
C_{0}=\frac{n_{A}^{0}}{n_{A}^{0}+n_{B}^{0}},
$$




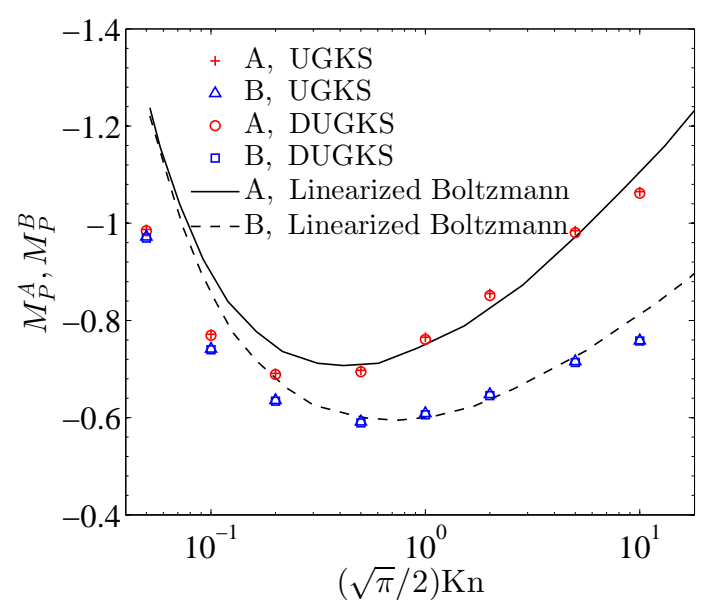

(a)

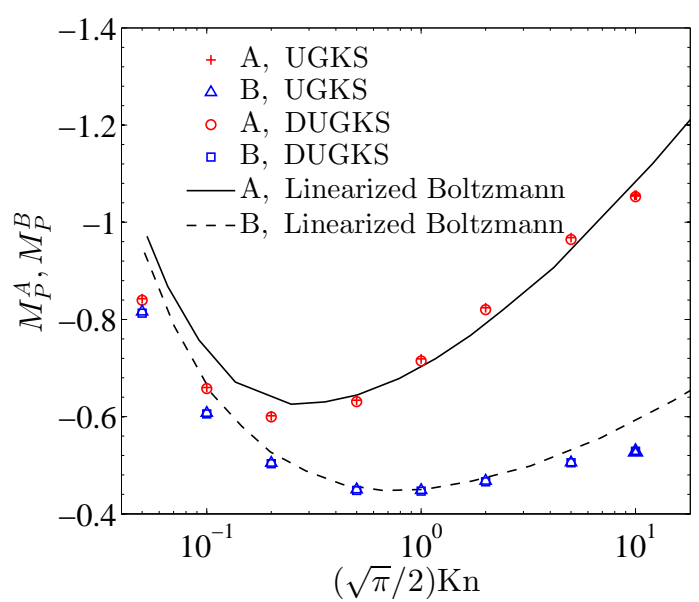

(b)

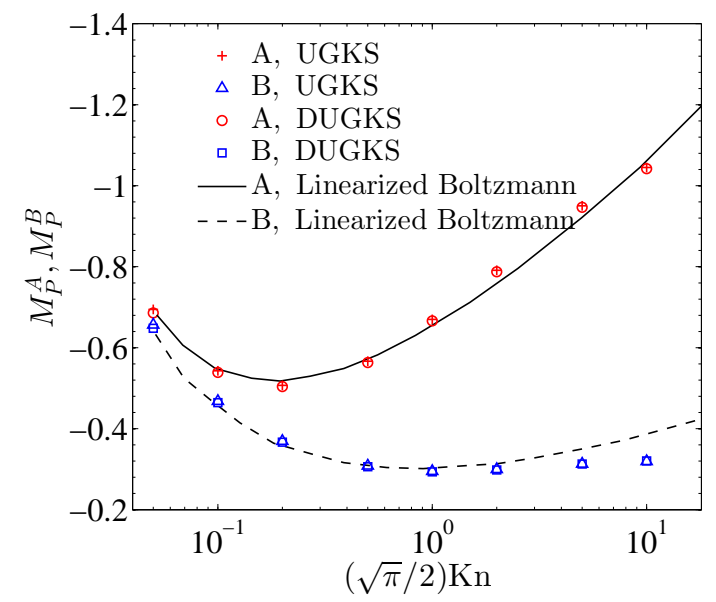

(c)

FIG. 5. Particle flux versus Knudsen number of the pressure driven channel flow with $\chi_{0}^{A}=0.5$. $m_{B} / m_{A}$ in (a), (b) and (c) are 2, 4 and 10, respectively. The linearized solutions are from Ref. [51].

with $n_{A}^{0}, n_{B}^{0}$ being the equilibrium number density of species $A$ and $B$, respectively. In this case, we focus on the shear stress $P_{x y}^{\prime}=P_{A x y}^{\prime}+P_{B x y}^{\prime}$ of the mixture and the velocity $u_{\alpha}^{\prime}$ of species $\alpha$. The shear stress $P_{\alpha x y}^{\prime}$ of species $\alpha$ is calculated according to Eq. (22). The velocity of the mixture in $x$-direction is defined as

$$
u_{m, x}=\frac{\rho_{A} u_{A, x}^{\prime}+\rho_{B} u_{B, x}^{\prime}}{\rho_{A}+\rho_{B}} .
$$

The gas rarefaction parameter $\delta$ is defined as

$$
\delta=\frac{H P_{0}}{\mu v_{0}}, \quad P_{0}=n_{0} k_{B} T_{0}
$$




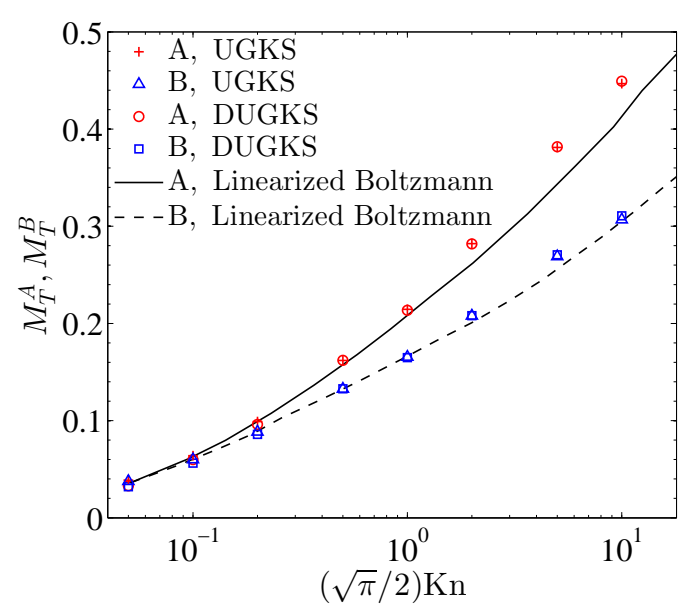

(a)

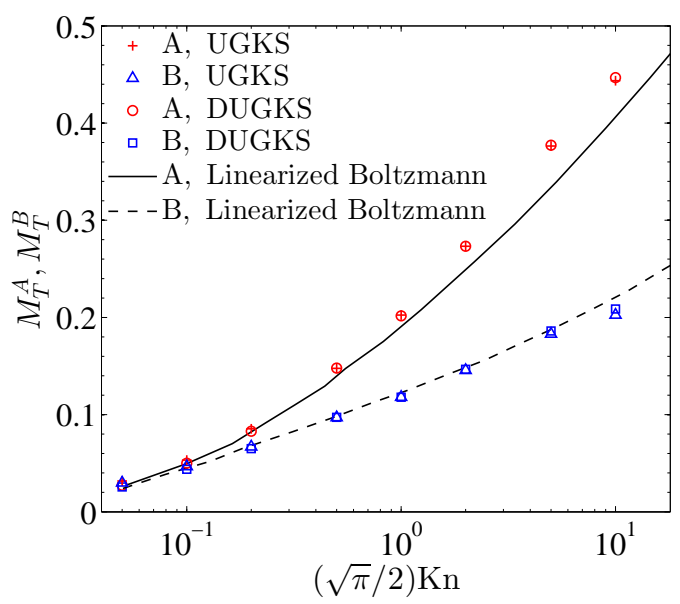

(b)

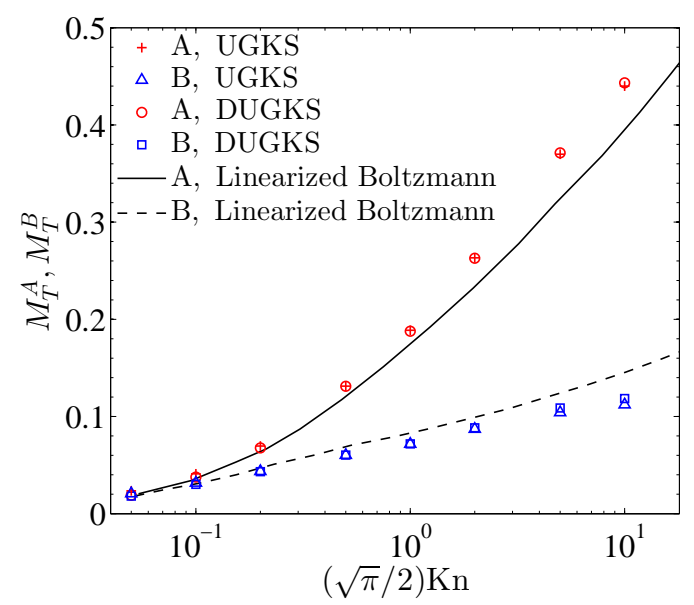

(c)

FIG. 6. Same as Fig. 5 except that the flow is driven by a temperature gradient.

where $\mu$ is the mixture viscosity at temperature $T_{0}$, and $P_{0}$ is the equilibrium pressure with $n_{0}$ being the total number density of the two species. Then we can get the following dimensionless quantities

$$
u_{\alpha}=\frac{u_{\alpha}^{\prime}}{U}, \quad P_{x y}=-\frac{v_{0}}{2 U P_{0}} P_{x y}^{\prime}
$$

To calculate $a_{B B} / a_{A A}$ by Eq. (17), the mass ratio and viscosity ratio should be known. Here we consider two groups of binary gas mixtures of noble gases: neon-argon (Ne-Ar) and helium-xenon (He-Xe). The molecular masses of these gases are $m_{H e}=4.0026, m_{N e}=$ 20.1791, $m_{A r}=39.948$ and $m_{X e}=131.293$ in atomic units. The experimental data [53] of the viscosities at an equilibrium temperature $T_{0}=300 \mathrm{~K}$ are $\mu_{\mathrm{He}}=19.73 \mu \mathrm{Pa} \cdot \mathrm{s}, \mu_{\mathrm{Ne}}=$ 


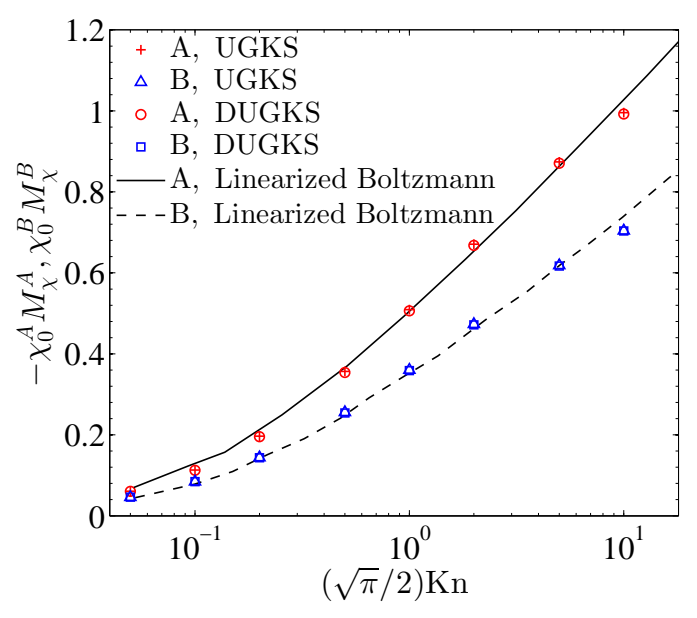

(a)

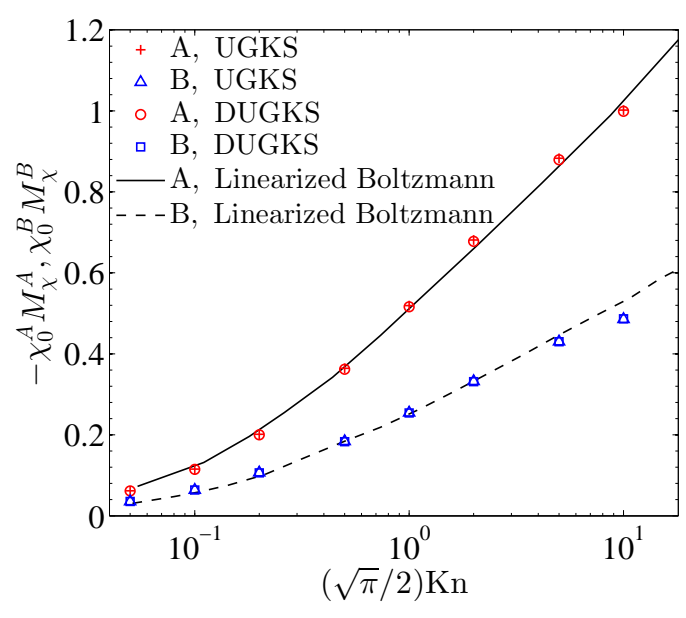

(b)

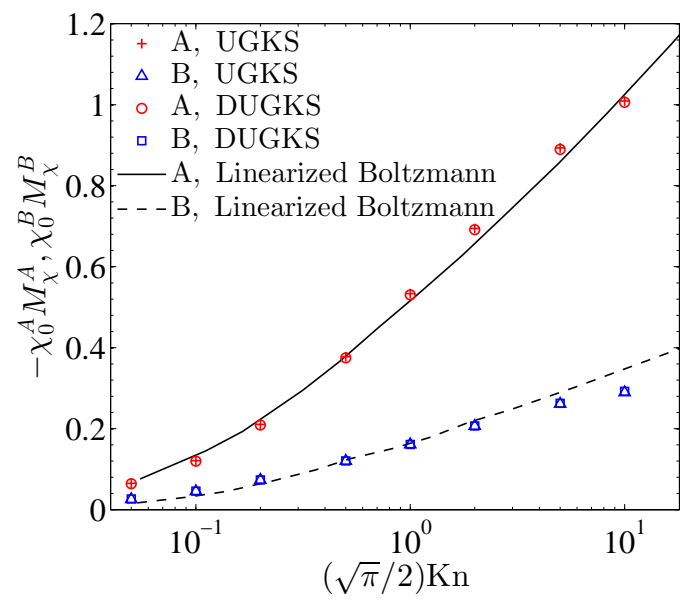

(c)

FIG. 7. Same as Fig. 5 except that the flow is driven by a concentration gradient.

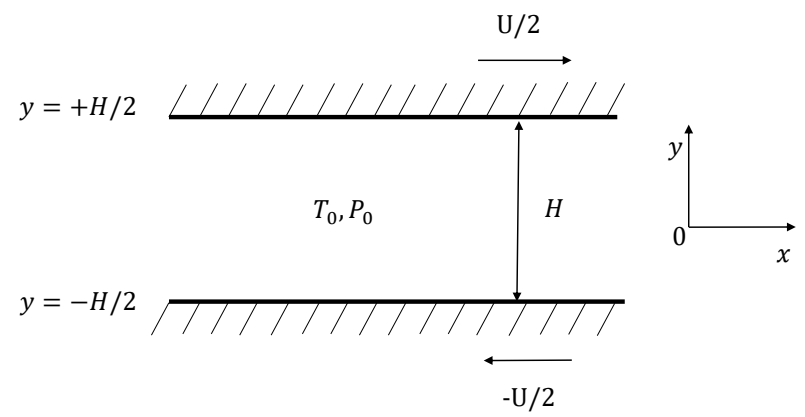

FIG. 8. Schematic of the plane Couette flow. 
$31.60 \mu \mathrm{Pa} \cdot s, \mu_{A r}=22.39 \mu \mathrm{Pa} \cdot s$ and $\mu_{X e}=22.62 \mu \mathrm{Pa} \cdot s$.

In our simulations, we take $U=0.1 v_{0}$ and the rarefaction parameter $\delta$ varies from 0.1 to 10 . Three values of $C_{0}=0.1,0.5$, and 0.9 are considered. The physical space is divided uniformly into 2 grid points in $x$-direction and 400 in $y$-direction. The half-range GaussHermite quadrature [54] with $28 \times 28$ velocity points is adopted for each species. The CFL number is set to be 0.6 in the following cases. In addition, the flow field is assumed to be steady when the maximum relative change of the velocity field of the two species in two successive steps is less than $10^{-10}$, i.e.,

$$
\int_{-1 / 2}^{1 / 2} \max \left(\frac{\left|u_{A, x}^{n+1}-u_{A, x}^{n}\right|}{\left|u_{A, x}^{n}\right|}, \frac{\left|u_{B, x}^{n+1}-u_{B, x}^{n}\right|}{\left|u_{B, x}^{n}\right|}\right) d y<10^{-10} .
$$

The results of the present DUGKS will be compared with the solutions of the McCormack model in Refs. [55] and [56]. The results of the UGKS for binary gas mixtures [28, 29] of Maxwell molecules are also presented for comparison. A comparative study between the McCormack model and the linearized Boltzmann equation for this problem was performed in Ref. [56]. The results indicate that differences in shear stress between the two models are small, while differences in other macroscopic quantities such as the velocity of each species are rather large, which increase with the mass ratio of the two species and the molar concentration of the heavy species.

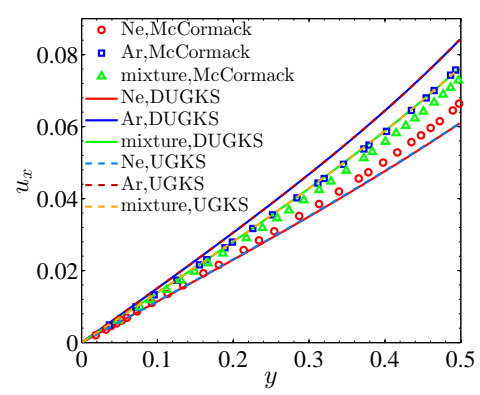

(a)

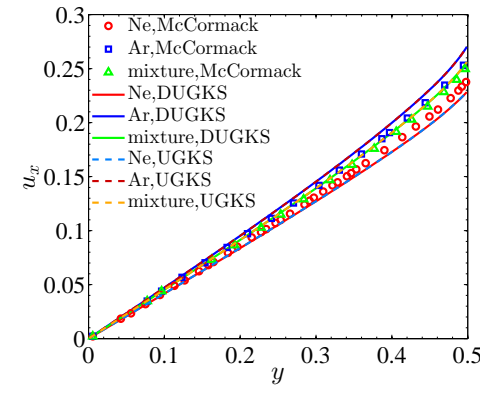

(b)

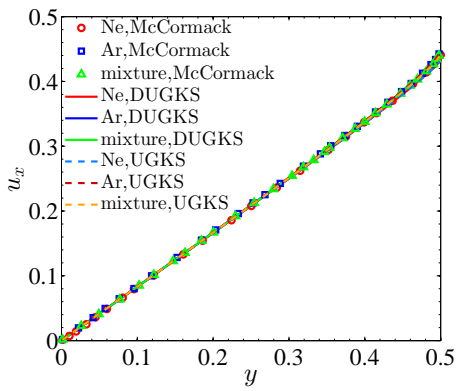

(c)

FIG. 9. Velocity profiles in the Couette flow for the Ne-Ar mixture with $C_{0}=0.5$ at (a) $\delta=0.1$, (b) $\delta=1$ and (c) $\delta=10$.

The velocity profiles for the Ne-Ar and He-Xe mixtures with molar concentration $C_{0}=0.5$ at $\delta=0.1,1$ and 10 are demonstrated in Fig. 9 and Fig. 10. It can be seen that the results predicted by the DUGKS agree well with those by the UGKS at different rarefaction 
TABLE I. Species velocity $u_{N e}$ and $u_{A r}$ and mixture velocity $u_{m}$ near the top plate $(y=H / 2)$ for the Ne-Ar mixture with concentration of the light species $C_{0}$ and rarefaction parameter $\delta$.

\begin{tabular}{|c|c|c|c|c|c|c|c|}
\hline \multirow[t]{2}{*}{$\delta$} & \multicolumn{2}{|r|}{$u_{N e}$} & \multicolumn{3}{|c|}{$u_{A r}$} & \multicolumn{2}{|r|}{$u_{m}$} \\
\hline & Present & UGKS Ref. [56] & Present & UGKS & Ref. [56] & Present & UGKS Ref. [56] \\
\hline \multicolumn{8}{|c|}{$C_{0}=0.1$} \\
\hline 0.1 & 0.0545 & $0.0546 \quad 0.0633$ & 0.0745 & 0.0753 & 0.0736 & 0.0737 & $0.0742 \quad 0.0730$ \\
\hline 1.0 & 0.2141 & $0.2141 \quad 0.2341$ & 0.2542 & 0.2543 & 0.2536 & 0.2521 & $0.2522 \quad 0.2525$ \\
\hline 10.0 & 0.4261 & $0.4261 \quad 0.4361$ & 0.4396 & 0.4397 & 0.4420 & 0.4384 & 0.43890 .4417 \\
\hline \multicolumn{8}{|c|}{$C_{0}=0.5$} \\
\hline 0.1 & 0.0609 & 0.06100 .0663 & 0.0844 & 0.0842 & 0.0773 & 0.0764 & 0.07640 .0736 \\
\hline 1.0 & 0.2280 & 0.22830 .2399 & 0.2699 & 0.2700 & 0.2598 & 0.2559 & $0.2560 \quad 0.2531$ \\
\hline 10.0 & 0.04310 & 0.43110 .4377 & 0.4437 & 0.4437 & 0.4435 & 0.4394 & 0.43950 .4416 \\
\hline \multicolumn{8}{|c|}{$C_{0}=0.9$} \\
\hline 0.1 & 0.0699 & $0.0700 \quad 0.0712$ & 0.0973 & 0.0976 & 0.0833 & 0.0749 & $0.0750 \quad 0.0734$ \\
\hline 1.0 & 0.2456 & $0.2457 \quad 0.2492$ & 0.2887 & 0.2889 & 0.2695 & 0.2534 & 0.25350 .2529 \\
\hline 10.0 & 0.4369 & 0.43690 .4407 & 0.4484 & 0.4485 & 0.4463 & 0.4389 & $0.4390 \quad 0.4417$ \\
\hline
\end{tabular}

TABLE II. The shear stresses in the Couette flow of the Ne-Ar and He-Xe mixtures under different rarefaction parameter $\delta$ and concentration of the light species $C_{0}$.

\begin{tabular}{|c|c|c|c|c|c|c|c|c|c|}
\hline \multirow[t]{2}{*}{$\delta$} & \multicolumn{3}{|c|}{$C_{0}=0.1$} & \multicolumn{3}{|c|}{$C_{0}=0.5$} & \multicolumn{3}{|c|}{$C_{0}=0.9$} \\
\hline & Present & UGKS & Ref. [55] & Present & UGKS & Ref. [55] & Present & UGKS & Ref. [55] \\
\hline \multicolumn{10}{|c|}{$\mathrm{Ne}-\mathrm{Ar}$} \\
\hline 0.1 & 0.2600 & 0.2600 & 0.2601 & 0.2568 & 0.2568 & 0.2576 & 0.2590 & 0.2590 & 0.2594 \\
\hline 1.0 & 0.1683 & 0.1684 & 0.1689 & 0.1657 & 0.1658 & 0.1675 & 0.1677 & 0.1678 & 0.1685 \\
\hline 10.0 & 0.04143 & 0.04145 & 0.04150 & 0.04115 & 0.04112 & 0.04139 & 0.04137 & 0.04142 & 0.04147 \\
\hline \multicolumn{10}{|c|}{ He-Xe } \\
\hline 0.1 & 0.2522 & 0.2523 & 0.2527 & 0.2111 & 0.2112 & 0.2163 & 0.1810 & 0.1811 & 0.1919 \\
\hline 1.0 & 0.1641 & 0.1642 & 0.1655 & 0.1337 & 0.1338 & 0.1482 & 0.1171 & 0.1171 & 0.1360 \\
\hline 10.0 & 0.04094 & 0.0410 & 0.04128 & 0.03645 & 0.03646 & 0.03999 & 0.03642 & 0.03643 & 0.03898 \\
\hline
\end{tabular}




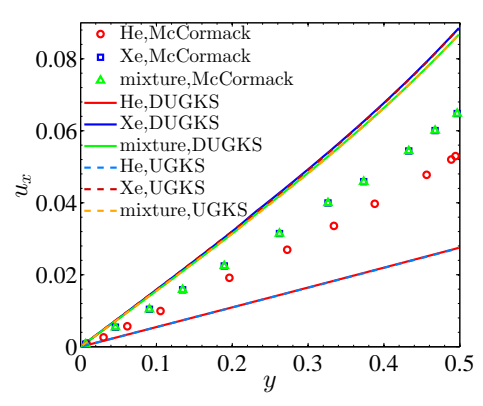

(a)

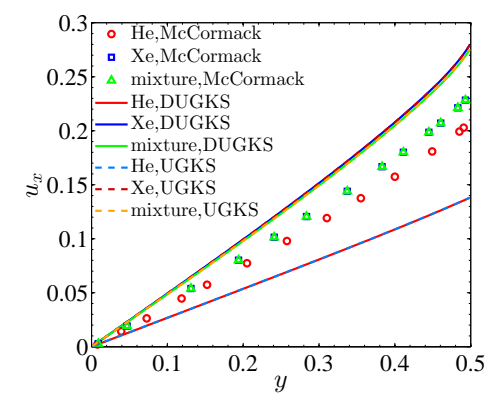

(b)

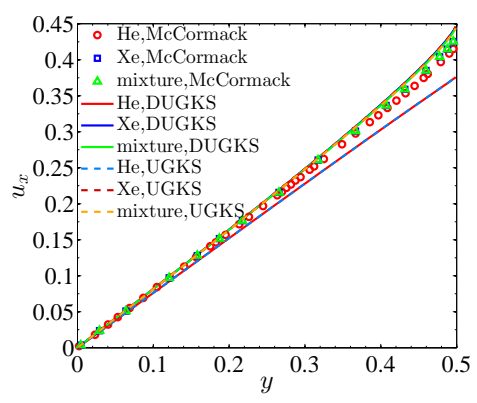

(c)

FIG. 10. Same as Fig. 9 but for the He-Xe mixture.

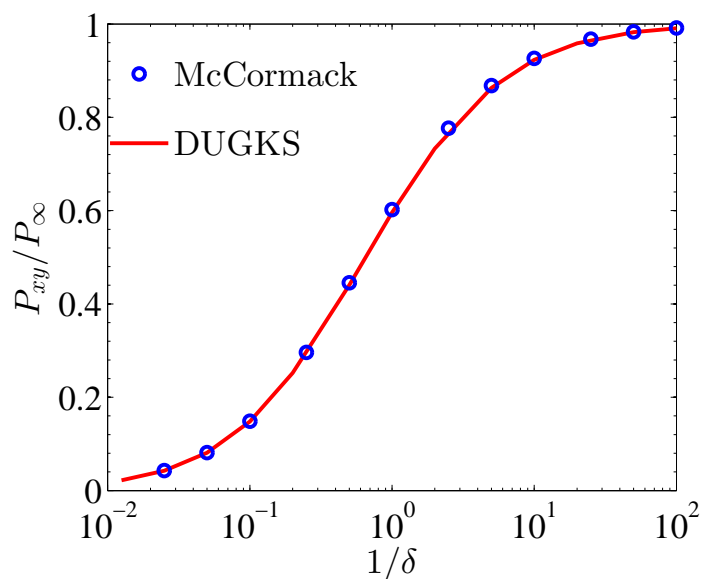

(a)

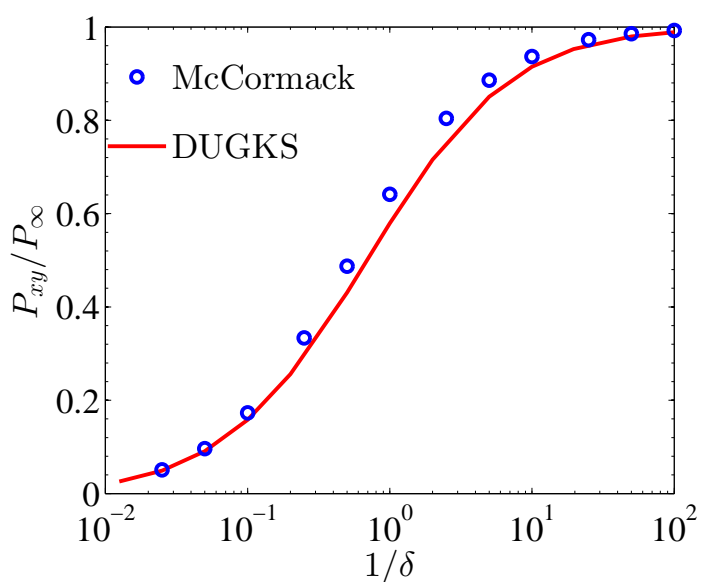

(b)

FIG. 11. The normalized stress of the Couette flow for gas mixtures (a) Ne-Ar and (b) He-Xe under different rarefaction parameter $\delta$ with $C_{0}=0.5$.

parameters. As $\delta=0.1$, the velocity differences of Ne and Ar between the DUGKS and the McCormack model are about $8 \%$ and $9 \%$, respectively. As $\delta=1$, the velocity differences decrease. As $\delta=10$, there are slightly differences in the velocity of the two species, i.e., $1.5 \%$ for Ne and $0.04 \%$ for Ar. The velocity of the He-Xe mixture is also shown in Fig. 10 to illustrate the influence of the mass ratio on the velocity. It can be seen that the deviations in velocity between the DUGKS and the McCormack model are significant, especially as $\delta$ is small. For instance, the velocity difference of He between the two models reaches $46.8 \%$ at $\delta=0.1$. Furthermore, the DUGKS overestimates the Xe velocity by $32.8 \%$ compared with the McCormack model. These differences decrease with increasing $\delta$, reducing to $10.6 \%$ for He and $2.97 \%$ for Xe as $\delta=10$. 
The influence of the molar concentration $C_{0}$ on the gas velocity near the plate is displayed in Table I. When $\delta=0.1$, the velocity difference of Ne between the two kinetic models decreases from $13.9 \%$ to $1.8 \%$, while that for Ar increases from $2 \%$ to $17 \%$ as $C_{0}$ increases from 0.1 to 0.9 . As $\delta=1$, the difference in each velocity is smaller than that for $\delta=0.1$ for all considered values of $C_{0}$. For $\delta=10$, the velocity difference of Ne between the DUGKS and the McCormack model is less than 2.3\% and that of $\mathrm{Ar}$ is less than $1 \%$ as $C_{0}$ varies from 0.1 to 0.9 .

The shear stresses $P_{x y}$ of the Ne-Ar and He-Xe mixtures are presented in Table II under different rarefaction parameters and molar concentrations. The shear stress of each mixture should be constant theoretically due to the momentum conservation of the mixture. While the numerical results can slightly deviate from the theoretical values. Thus the average shear stress $P_{x y}^{a v}=\int_{-1 / 2}^{1 / 2} P_{x y}(y) d y$ is presented here. The maximum variation of the shear stress is calculated as

$$
\Delta P_{x y}=\max _{y \in\left[-\frac{H}{2}, \frac{H}{2}\right]}\left\|\frac{P_{x y}(y)-P_{x y}^{a v}}{P_{x y}^{a v}}\right\|,
$$

which is less than $0.7 \%$ according to Eq. (57), showing a good numerical accuracy of the proposed DUGKS. In the Ne-Ar mixture, the differences in shear stress between the results obtained from the DUGKS and the McCormack model are less than $1 \%$ for all values of the considered molar concentration $C_{0}$ and rarefaction $\delta$, meaning a good agreement between these two kinetic models at small mass ratio. In the He-Xe mixture whose mass ratio is much greater than that of the Ne-Ar mixture, the relative differences between results from the two kinetic models are less than $1 \%$ for all considered values of $C_{0}$ as $\delta=0.01$ and 40. However, the DUGKS underpredicts the shear stress by $2.4 \%$ as $C_{0}=0.5$ and $5.6 \%$ as $C_{0}=0.9$ when $\delta=0.1$ for the He-Xe mixture. As $\delta=1$, the differences increase and reach to $9.7 \%$ for $C_{0}=0.5$ and $13.8 \%$ for $C_{0}=0.9$. For $\delta=10$, the differences in shear stress are $8.9 \%$ and $6.5 \%$ for $C_{0}=0.5$ and $C_{0}=0.9$, respectively. These comparisons indicate that the relative difference in shear stress of the He-Xe mixture between the DUGKS and the McCormack model is much higher than that of the Ne-Ar mixture, especially for $\delta=1$, at which the difference reaches the maximum.

The influence of the rarefaction parameter $\delta$ on the shear stress is demonstrated in Fig. 11 with $\delta$ ranging from 0.01 to 80 and $C_{0}=0.5$ for the Ne-Ar and He-Xe mixtures. It shows a good agreement between the DUGKS and the McCormack model over the whole range of 
the flow regime for the Ne-Ar mixture. While for the He-Xe mixture, clear deviations are observed in the slip and transitional regimes between the two models, suggesting that the difference between the two models increases with mass ratio.

\section{Lid-driven cavity flow}

The last test case is the two-dimensional lid-driven cavity flow of binary gas mixtures. The flow domain is a square cavity with length $H$ and the upper wall moves with a constant velocity $U_{w}$ which is also much smaller than the characteristics molecular velocity $v_{0}$ in Eq. (51), while other walls are fixed. The temperature at the four walls is fixed at $T_{0}$, and the walls are fully diffusive. The Ne-Ar and He-Xe mixtures are considered to investigate the influence of the mass ratio on the mixture velocity and temperature. We also simulate this problem by the UGKS [28, 29] and the DSMC [57] to validate the current DUGKS method.

Firstly, to verify the indifferentiability principle of the AAP model, we set $m_{A} / m_{B}=1$, $\mu_{A} / \mu_{B}=1$, and $a_{A A} / a_{B B}=1$, namely the mixture reduces to a single-species gas. The rarefaction parameter $\delta$ is related to the Reynolds number as

$$
\delta=\frac{\operatorname{Re} k_{B} T_{0}}{m_{A} U_{w} v_{0}}
$$

where

$$
\operatorname{Re}=\frac{\rho_{0} U_{w} H}{\mu_{A}}, \quad \rho_{0}=n_{0} m_{A} .
$$

In the simulations, we take $U_{w}=0.1 v_{0}$. The velocity space is discretized via the half-range Gauss-Hermite quadrature with $8 \times 8$ velocity nodes. The physical space is divided into $120 \times 120$ uniform cells. The CFL number is set to be 0.6 . Figure 12 shows the velocity profiles along the centerlines of the cavity at $\mathrm{Re}=400$. The Ghia's benchmark solutions [58] are also included for comparison. It can be seen that the DUGKS results agree well with the benchmark data, suggesting that the AAP model satisfies the indifferentiability principle, which requires that the total distribution function $f=\sum_{\alpha=A, B} f_{\alpha}$ satisfies the single-species BGK equation when the two species are the same. Besides, the ratio of the time step to the mean collision time $(\Delta t / \tau)$ is about 3.146 for this case $\left(\mathrm{Kn} \approx 5 \times 10^{-4}\right.$ ), which clearly

shows that the time step of the DUGKS is not limited by the mean collision time. Thus the AP property of the DUGKS for the Navier-Stokes limit is validated. 


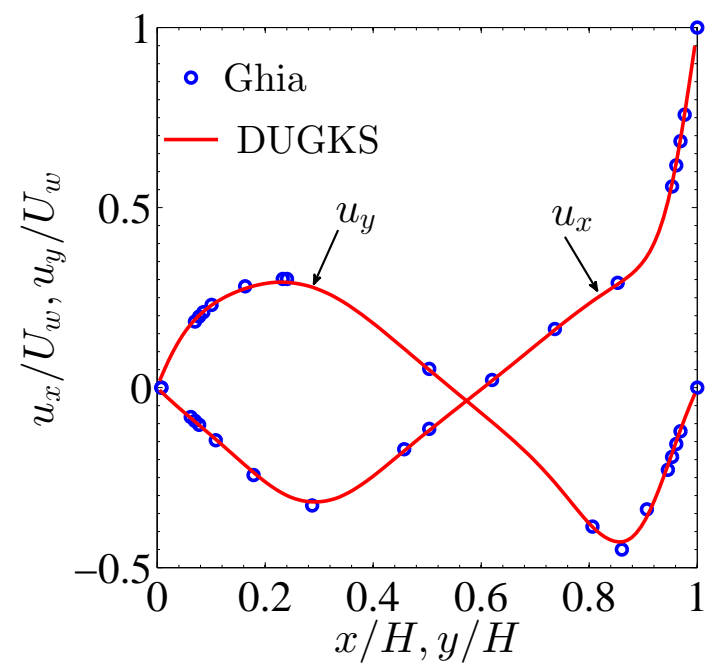

FIG. 12. Velocity profiles across the cavity center at $\operatorname{Re}=400$.

Then the flows of two groups of binary gas mixtures (Ne-Ar and He-Xe) are simulated. The velocity space for each species is discretized using the Newton-Cotes rule, with $101 \times 101$ velocity points distributed uniformly in $\left[-4 \sqrt{2 R_{\alpha} T_{0}}, 4 \sqrt{2 R_{\alpha} T_{0}}\right] \times\left[-4 \sqrt{2 R_{\alpha} T_{0}}, 4 \sqrt{2 R_{\alpha} T_{0}}\right]$. The physical space is divided into $60 \times 60$ cells uniformly, where the results are nearly identical to those on a $100 \times 100$ mesh.

The velocity profiles of the two mixtures across the cavity center for $\delta=0.1,1$ and 10 under the concentration of the light species $C_{0}=0.5$ are presented in Figs. 13 and 14. Good agreement can be observed between the DUGKS and the UGKS and DSMC results for the Ne-Ar mixture as $\delta$ varies from 0.1 to 10 . However, for the He-Xe mixture, whose molecular mass ratio is large, deviations between the solutions of DSMC and the DUGKS increase with $\delta$. This discrepancy can be attributed to the relaxation approximation of the collision operator. The above comparisons show that the DUGKS based on the AAP model can offer accurate flow solutions for flows in the rarefied regimes, as the molecular mass ratio is not large.

Similar phenomena can also be found in the temperature field as shown in Figs. 15 and 16. The translational kinetic temperature [6] is considered in this case, which is defined by

$$
\frac{3}{2} k_{B} T_{t r}=\frac{1}{2} \sum_{\alpha}\left(n_{\alpha} / n\right) m_{\alpha} \overline{c_{\alpha}^{\prime 2}},
$$

where $\overline{c_{\alpha}^{\prime 2}}$ can be expressed as

$$
\overline{c_{\alpha}^{\prime 2}}=u_{\alpha}^{2}+3 R_{\alpha} T_{\alpha}-u_{m}^{2} .
$$


For the Ne-Ar mixture with a small mass ratio, the DUGKS results agree excellently with the DSMC solutions in all cases. For the He-Xe mixture with a large mass ratio, the results of DUGKS and UGKS always have good agreement, while they deviate from the DSMC solutions for large $\delta$.

In order to evaluate the computational efficiency, we also measure the computing time of the DUGKS, UGKS and the DSMC method using the lid-driven cavity flow case with of the Ne-Ar mixture. Both the DUGKS and UGKS are run with 24 cores using OpenMP programming, while the DSMC solver is run with 48 cores using MPI programming. The wall time (in seconds) and numbers of iterations to reach the steady states in DUGKS/UGKS and the current noise level in DSMC for $\delta=0.1,1$ and 10 are listed in Table III. We can see the DUGKS is about $15 \%$ to $64 \%$ faster than UGKS depending on the rarefaction degree and is significantly faster than DSMC method.

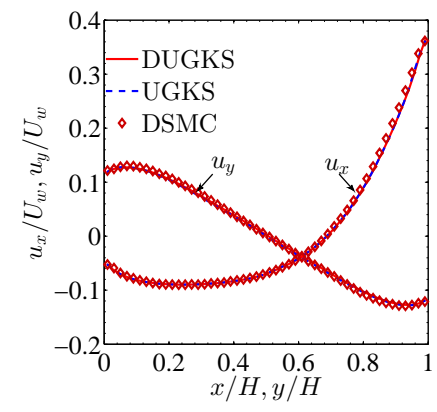

(a)

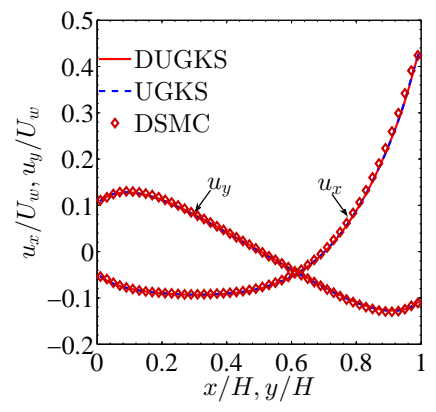

(b)

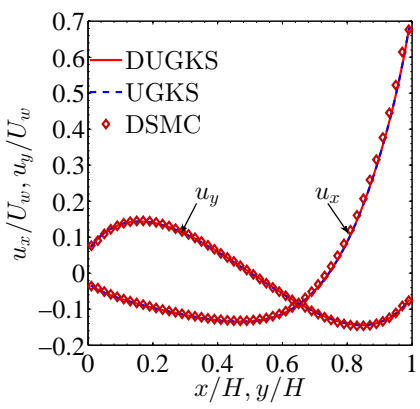

(c)

FIG. 13. Velocity profiles along the center lines in the cavity flow for the Ne-Ar mixture with $C_{0}=0.5$ at (a) $\delta=0.1$, (b) $\delta=1$ and (c) $\delta=10$.

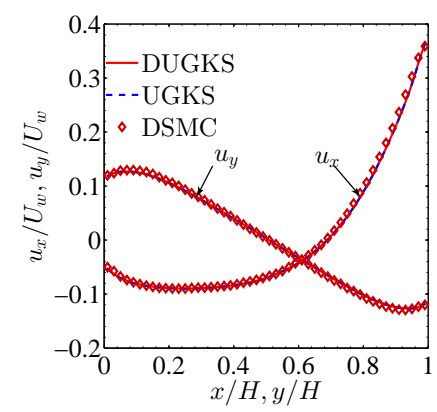

(a)

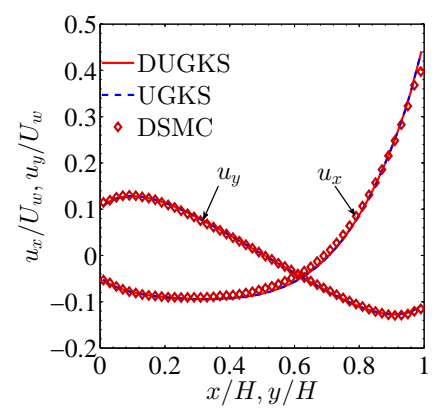

(b)

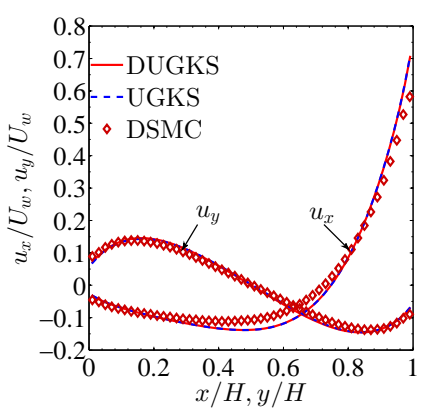

(c)

FIG. 14. Same as Fig. 13 but for the He-Xe mixture. 


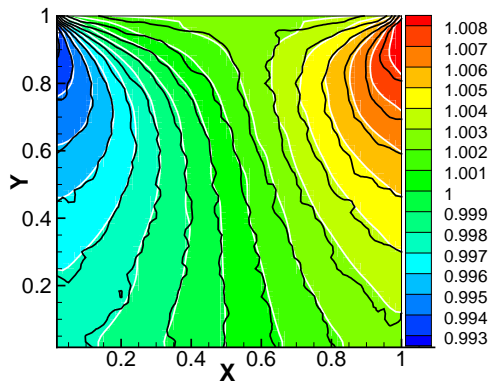

(a)

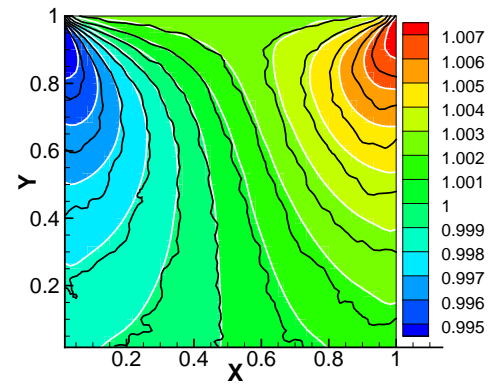

(b)

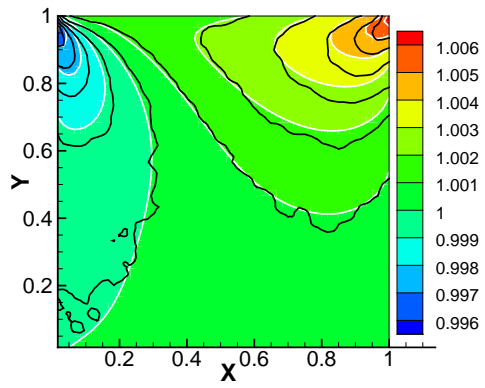

(c)

FIG. 15. Temperature contours in the lid driven cavity flow for the Ne-Ar mixture with $C_{0}=0.5$ at (a) $\delta=0.1$, (b) $\delta=1$ and (c) $\delta=10$. Black line: DSMC; white line with background: DUGKS.

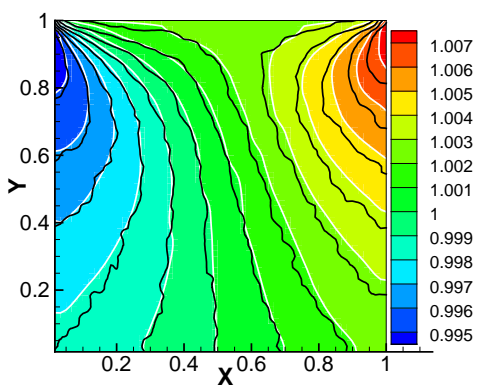

(a)

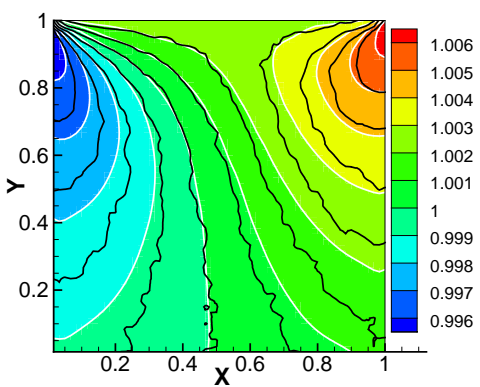

(b)

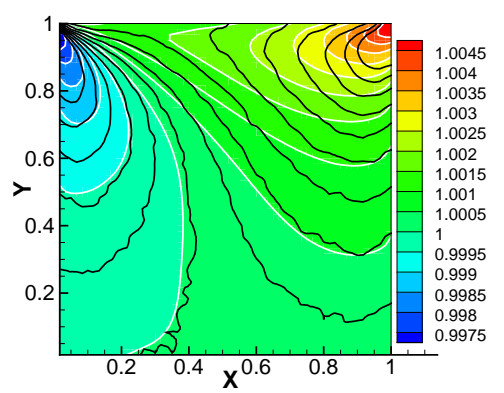

(c)

FIG. 16. Same as Fig. 15 but for the He-Xe mixture.

\section{CONCLUSIONS}

In this paper, a DUGKS is developed for flows of binary gas mixtures of Maxwell molecules in the whole range of the Knudsen number based on the AAP model. The numerical scheme possesses the asymptotic preserving (AP) property, which means that the time step and the cell size are not constrained by the particle collision time and the mean free path of gas molecules respectively when solving the Navier-stokes equations at the continuum limit.

In order to validate the DUGKS, several tests have been performed, including the shock structure, the channel flows driven by a small gradient of pressure, or temperature, or concentration, the plane Couette flow and the cavity flow in all flow regimes. Excellent agreement has been obtained between the solutions of the DUGKS and the UGKS for all cases, and good agreement with the reference solutions is obtained at moderate Knudsen numbers and 
TABLE III. Wall time (in seconds) and iteration steps of reaching steady states. 24 cores are used in the DUGKS and UGKS, and 48 cores are used in DSMC.

\begin{tabular}{|c|c|c|c|c|c|c|}
\hline \multirow[t]{2}{*}{$\delta$} & \multicolumn{2}{|c|}{ DUGKS } & \multicolumn{2}{|c|}{ UGKS } & \multicolumn{2}{|c|}{ DSMC } \\
\hline & time & step & time & step & time & step \\
\hline 0.1 & 11454 & 17217 & 18787 & 19880 & 37534 & $6,110,000$ \\
\hline 1 & 7240 & 9625 & 8314 & 8770 & 58244 & $10,000,000$ \\
\hline 10 & 10470 & 14516 & 15633 & 16490 & 92043 & $10,000,000$ \\
\hline
\end{tabular}

mass ratios in channel flow, especially for the heavy species. But some deviations in temperature are found for the light species, which can be attributed to the incorrect Prandt number of AAP model. For the plane Couette flow, the DUGKS results agree well with those of the McCormack model at small mass ratio and small Knudsen numbers, otherwise obvious differences are observed. As for the cavity flow, the proposed DUGKS results agree well with the DSMC solutions in all flow regimes when the mass ratio is small, but clear deviations appear in the near-continuum regime with large mass ratio, which can be attributed to the relaxation approximation of the collision operator.

Finally, it should be pointed out that the present DUGKS is based on the AAP model for Maxwell molecular gases, which has its own limitations as indicated in the simulation results. Further development of the method based on more accurate kinetic models such as the ellipsoidal models $[59,60]$ or the McCormack model will be studied in the future work.

\section{ACKNOWLEDGMENTS}

This study is financially supported by the National Key Research and Development Plan (No. 2016YFB0600805) and the National Natural Science Foundation of China (No. 11702223). 


\section{REFERENCES}

[1] F. Sharipov, Rarefied gas dynamics: fundamentals for research and practice (John Wiley \& Sons, 2015).

[2] G. Karniadakis, A. Beskok, and M. Gad-El-Hak, Applied Mechanics Reviews 55, B76 (2002).

[3] O. Aktas, N. Aluru, and U. Ravaioli, Journal of Microelectromechanical Systems 10, 538 (2001).

[4] F. Sharipov and J. L. Strapasson, Physics of Fluids 25, 027101 (2013).

[5] L. Szalmas, D. Valougeorgis, and S. Colin, in Proceedings of ASME 2011 9th International Conference on Nanochannels, Microchannels and Minichannels, Vol. 1 (2011) pp. 279-288.

[6] G. A. Bird, Molecular Gas Dynamics and the Direct Simulation of Gas Flows (Clarendon Press, 1994).

[7] T. M. Homolle and N. G. Hadjiconstantinou, Journal of Computational Physics 226, 2341 (2007).

[8] L. Szalmas, Journal of Computational Physics 231, 3723 (2012).

[9] S. Kosuge, K. Aoki, and S. Takata, European Journal of Mechanics-B/Fluids 20, 87 (2001).

[10] S. Kosuge, K. Aoki, and S. Takata, in AIP Conference Proceedings, Vol. 585 (AIP, 2001) pp. 289-296.

[11] R. Garcia and C. Siewert, European Journal of Mechanics-B/Fluids 26, 749 (2007).

[12] R. Garcia and C. Siewert, European Journal of Mechanics-B/Fluids 27, 823 (2008).

[13] F. J. McCormack, The Physics of Fluids 16, 2095 (1973).

[14] S. Kosuge, European Journal of Mechanics-B/Fluids 28, 170 (2009).

[15] C. Tantos and D. Valougeorgis, International Journal of Heat and Mass Transfer 117, 846 (2018).

[16] P. Andries, K. Aoki, and B. Perthame, Journal of Statistical Physics 106, 993 (2002).

[17] P. L. Bhatnagar, E. P. Gross, and M. Krook, Phys. Rev. 94, 511 (1954).

[18] M. Groppi and G. Spiga, Physics of Fluids 16, 4273 (2004).

[19] M. Groppi, K. Aoki, G. Spiga, and V. Tritsch, Physics of Fluids 20, 117103 (2008).

[20] C. Liu and K. Xu, Communications in Computational Physics 22, 1175 (2017). 
[21] M. Bisi and S. Lorenzani, Physics of Fluids 28, 052003 (2016).

[22] L. Szalmas, Open Physics 6, 786 (2008).

[23] P. Asinari and L.-S. Luo, Journal of Computational Physics 227, 3878 (2008).

[24] Z. Guo, P. Asinari, and C. Zheng, Physics Review E 79, 026702 (2009).

[25] F. Sharipov and D. Kalempa, Journal of Vacuum Science \& Technology A: Vacuum, Surfaces, and Films 20, 814 (2002).

[26] S. Naris, D. Valougeorgis, F. Sharipov, and D. Kalempa, Superlattices and Microstructures 35, 629 (2004).

[27] D. Valougeorgis and S. Naris, SIAM Journal on Scientific Computing 25, 534 (2003).

[28] R. Wang, Unified gas-kinetic scheme for the study of non-equilibrium flows, Ph.D. thesis, Hong Kong University of Science and Technology (2015).

[29] R. Wang and K. Xu, in AIP Conference Proceedings, Vol. 1628.

[30] K. Xu and J.-C. Huang, Journal of Computational Physics 229, 7747 (2010).

[31] J.-C. Huang, K. Xu, and P. Yu, Communications in Computational Physics 12, 662 (2012).

[32] L. Mieussens, Journal of Computational Physics 253, 138 (2013).

[33] Z. Guo, K. Xu, and R. Wang, Physics Review E 88, 033305 (2013).

[34] Z. Guo, R. Wang, and K. Xu, Physics Review E 91, 033313 (2015).

[35] P. Wang, L. Zhu, Z. Guo, and K. Xu, Communications in Computational Physics 17, 657 (2015).

[36] P. Wang, S. Tao, and Z. Guo, Computers \& Fluids 120, 70 (2015).

[37] L. Zhu, Z. Guo, and K. Xu, Computers \& Fluids 127, 211 (2016).

[38] L. Zhu and Z. Guo, Physics Review E 95, 023113 (2017).

[39] Z. Guo and K. Xu, International Journal of Heat and Mass Transfer 102, 944 (2016).

[40] C. Zhang, K. Yang, and Z. Guo, arXiv preprint arXiv:1802.02868 (2018).

[41] M. Icardi, G. Boccardo, D. L. Marchisio, T. Tosco, and R. Sethi, Phys. Rev. E 90, 013032 (2014).

[42] T. W. Willingham, C. J. Werth, and A. J. Valocchi, Environmental Science \& Technology 42, 3185 (2008).

[43] G. M. Kremer, An introduction to the Boltzmann equation and transport processes in gases (Springer Science \& Business Media, 2010).

[44] T. Morse, The Physics of Fluids 6, 1420 (1963). 
[45] J. Yang and J. Huang, Journal of Computational Physics 120, 323 (1995).

[46] C. K. Chu, The Physics of Fluids 8, 12 (1964).

[47] B. Van Leer, Journal of computational physics 23, 276 (1977).

[48] S. Harris, An introduction to the theory of the Boltzmann equation (Courier Corporation, 2004).

[49] C. Cercignani, Mathematical methods in kinetic theory (Springer, 1969).

[50] G. Bird, Journal of Fluid Mechanics 31, 657 (1968).

[51] S. Kosuge and S. Takata, European Journal of Mechanics-B/Fluids 27, 444 (2008).

[52] C. Cercignani, in The Boltzmann Equation and Its Applications (Springer, 1988) pp. 40-103.

[53] J. Kestin, K. Knierim, E. Mason, B. Najafi, S. Ro, and M. Waldman, Journal of physical and chemical reference data 13, 229 (1984).

[54] B. Shizgal, Journal of Computational Physics 41, 309 (1981).

[55] F. Sharipov, L. M. G. Cumin, and D. Kalempa, European Journal of Mechanics-B/Fluids 23, 899 (2004).

[56] M. T. Ho, L. Wu, I. Graur, Y. Zhang, and J. M. Reese, International Journal of Heat and Mass Transfer 96, 29 (2016).

[57] T. Scanlon, E. Roohi, C. White, M. Darbandi, and J. Reese, Computers \& Fluids 39, 2078 (2010).

[58] U. Ghia, K. N. Ghia, and C. Shin, Journal of Computational Physics 48, 387 (1982).

[59] M. Groppi, S. Monica, and G. Spiga, EPL (Europhysics Letters) 96, 64002 (2011).

[60] S. Brull, V. Pavan, and J. Schneider, European Journal of Mechanics-B/Fluids 33, 74 (2012). 